\title{
BOUNDS OF GELFAND-TSETLIN MULTIPLICITIES AND TABLEAUX REALIZATIONS OF VERMA MODULES
}

\author{
VYACHESLAV FUTORNY, DIMITAR GRANTCHAROV, LUIS ENRIQUE RAMIREZ, \\ AND PABLO ZADUNAISKY
}

\begin{abstract}
Aвstract. We introduce the notion of essential support of a simple Gelfand-Tsetlin $\mathfrak{g l}_{n}$-module as an attempt towards understanding the character formula of such module. This support detects the weights in the module having maximal possible Gelfand-Tsetlin multiplicities. Using combinatorial tools we describe the essential supports of the simple socles of the universal tableaux modules. We also prove that every simple Verma module appears as the socle of a universal tableaux module. As a consequence, we prove the Strong Futorny-Ovsienko Conjecture on the sharpness of the upper bounds of the Gelfand-Tsetlin multiplicities. We also give a very explicit description of the support and essential support of the simple singular Verma module $M(-\rho)$.
\end{abstract}

MSC 2010 Classification: 16G99, 17B10.

Keywords: Gelfand-Tsetlin modules, Gelfand-Tsetlin bases, reflection groups, Verma modules.

\section{Introduction}

Gelfand-Tsetlin modules of the complex general linear Lie algebra $\mathfrak{g l}(n, \mathbb{C})$ have been studied since the 1950's both by mathematicians and physicists. These modules admit a locally finite action of the Gelfand-Tsetlin subalgebra $\Gamma$, a maximal commutative subalgebra of the universal enveloping algebra $U(\mathfrak{g l}(n))$. The recent discovery of Gelfand-Tsetlin derivative tableaux in [FGR16] initiated the systematic study of singular Gelfand-Tsetlin modules. This theory attracted considerable attention in the last three years and many interesting and important results have been obtained in [EMV, FGR17, FGR17b, FGRZ18, FRZ19, Har17, MV, RZ18, Vis, Vis18, Zad17]. Singular Gelfand-Tsetlin modules turned out to be related to different but overlapping theories. For example, connections with Schubert calculus were discovered in [FGRZ18] and with tensor product categorifications and KLRW algebras in $\left[\mathrm{KTW}^{+} 18\right]$. With the aid of KLRW algebras, in $\left[\mathrm{KTW}^{+}{ }_{18}\right]$, the authors provide a bijection between the set of simple Gelfand-Tsetlin $\mathfrak{g l}(n, \mathbb{C})$-modules with a fixed character and the zero weight space of an $\mathfrak{s l}(n, \mathbb{C})$-crystal. Furthermore, the properties of the singular Gelfand-Tsetlin modules have been studied with combinatorial 
tools, [FGR16, FGRZ18, FRZ19, RZ18], as well as with geometric methods, [EMV, MV, Vis18].

A maximal ideal $\mathfrak{m}$ of $\Gamma$ defines a point $v=v_{\mathfrak{m}}$ in $\mathbb{C}^{\mu}=\mathbb{C}^{1} \times \mathbb{C}^{2} \times \cdots \times$ $\mathbb{C}^{n}$ up to a permutation of coordinates. Given such $v$ one constructs a "universal" tableaux Gelfand-Tsetlin module $V(T(v))$ which contains a simple Gelfand-Tsetlin subquotient $M$ having $\mathfrak{m}$ in its support, i.e. $M[\mathfrak{m}] \neq 0$ (see \$3.1 for precise definitions). It was shown in [EMV, RZ18] that $V(T(v)$ ) has a basis of derivative tableaux, and the action of $\mathfrak{g l}(n, \mathbb{C})$ on this basis was described in [FGRZ18] in terms of BGG differential operators and Postnikov-Stanley polynomials. We conjecture that the module $V(T(v))$ is universal in the sense that every simple Gelfand-Tsetlin module having $\mathfrak{m}$ in its support is a subquotient of $V\left(T\left(v_{\mathfrak{m}}\right)\right)$. This conjecture was proven for generic $v$ in [FGR15] and for 1-singular $v$ in [FGR16, FGR17b].

In the present paper we make a significant step in the understanding of the structure of $V(T(v))$, in particular its socle. As a generating vector we choose a special vector $\bar{v} \in \mathbb{C}^{\mu}$, called a seed (see Definition 2.2), and show that the module $V(T(v))=V(T(\bar{v}))$ has a simple socle $V_{\text {soc }}$ whose structure can be described in terms of certain oriented graphs. The simple module $V_{\mathrm{soc}}$ is also a Gelfand-Tsetlin module such that $V_{\mathrm{soc}}=\bigoplus_{z} V_{\mathrm{soc}}[\bar{v}+z]$ where the sum is taken over a certain set of points of $\mathbb{C}^{\mu}$ with integral coordinates. The dimensions of the weight spaces $V_{\text {soc }}[\bar{v}+z]$ (called GelfandTsetlin multiplicities) are finite and uniformly bounded as explained in more detail below.

Set $S_{\mu}:=S_{1} \times \cdots \times S_{n}$ and consider the free abelian group $\mathbb{Z}_{0}^{\mu}$ consisting of elements in $\mathbb{C}^{\mu}$ with integer coordinates the last $n$ of which equal zero. Denote by GT the category of all Gelfand-Tsetlin $\mathfrak{g l}(n, \mathbb{C})$-modules, and for each equivalence class $\zeta \in \mathbb{C}^{\mu} /\left(\mathbb{Z}_{0}^{\mu} \# S_{\mu}\right)$ denote by $\mathrm{GT}_{\zeta}$ the full subcategory of GT consisting of modules whose support is contained in $\zeta$. We have a decomposition of GT into a direct sum of components

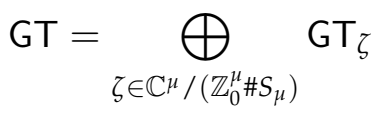

in the sense that $\operatorname{Ext}_{\mathrm{GT}}^{i}(M, N)=0$ for all $i \geq 0$ and for any $M$ and $N$ in different components (see [FO14, Corollary 3.4]).

An upper bound for the Gelfand-Tsetlin multiplicities of any simple Gelfand-Tsetlin module was found in [FO14, Theorem 4.12(c)]. To write this bound, fix a seed $\bar{v}$ and consider the stabilizer $S_{\pi(\bar{v})}$ of $\bar{v}$ in $S_{\mu}$. Let $z \in \mathbb{Z}_{0}^{\mu}$ be such that $\bar{v}+z$ is in normal form (see Definition 2.1) and let $\left(S_{\pi(\bar{v})}\right)_{z}$ be the stabilizer of $z$ in $S_{\pi(\bar{v})}$. Set $\zeta=\zeta_{\bar{v}}=\mathbb{C}^{\mu} /\left(\mathbb{Z}_{0}^{\mu} \# S_{\mu}\right) \bar{v}$. Then, as shown in [FO14], for any simple module $M$ in $\mathrm{GT}_{\zeta}$ the upper bound on 
a Gelfand-Tsetlin multiplicity is given by

(1) $\quad \operatorname{dim} M[\bar{v}+z] \leq \frac{\left|S_{\pi(\bar{v})}\right|}{\left|\left(S_{\pi(\bar{v})}\right)_{z}\right|}$.

We will refer to (11) as the FO inequality. In [FO14, Remark 5.4] Futorny and Ovsienko conjectured that this inequality is sharp, and more precisely, that there is a simple module $M$ for which equality holds in (1) for some $z$ with trivial stabilizer. This conjecture follows from either [FGRZ18, Theorems 8.3, 8.5] or [EMV], Theorems 10,11] for $z$ with any stabilizer. In fact, the results in [EMV] and [FGRZ18] imply that (1) holds for $M=V(T(\bar{v}))$. In the special case where none of the differences between entries in consecutive rows of $\bar{v}$ are integers, $V(T(\bar{v}))$ is simple.

In this paper we prove a stronger result, which we call the Strong FutornyOvsienko Conjecture. We show that the socle of $V(T(\bar{v}))$ is simple, and that taking $M$ to be this socle, the set of all $z \in \mathbb{Z}_{0}^{\mu}$ such that equality holds in (1) (the essential support of $M$ ) is a union of rational polyhedral cones, at least one of which has dimension $n(n-1) / 2$. We also show that the subgroups $\left(S_{\pi(\bar{v})}\right)_{z}$ run over all parabolic subgroups of $S_{\pi(\bar{v})}$. This shows that the FO inequality gives a sharp bound in each subcategory $\mathrm{GT}_{\zeta}$.

In the following theorem we summarize the above discussed results.

Theorem 1.1. Let $\bar{v}$ be a seed in $\mathbb{C}^{\mu}$ and $\zeta=\zeta_{\bar{v}}$. Then the following hold.

(i) The module $V(T(\bar{v}))$ has a simple socle $V_{\text {soc. }}$.

(ii) The Strong Futorny-Ovsienko Conjecture holds for $V_{\mathrm{soc}}$, i.e. the essential support of $V_{\text {soc }}$ is nonempty. Moreover, this essential support consists of the integral points of a finite union of polyhedral rational cones, at least one of which is of maximal possible rank, $\frac{n(n-1)}{2}$.

(iii) The maximal Gelfand-Tsetlin multiplicity of a character in $\mathrm{GT}_{\zeta}$ is $\left|S_{\pi(\bar{v})}\right|$, and this is attained at the socle $V_{\mathrm{soc}}$.

(iv) For any $\bar{v}+z$ in the essential support of $V_{\mathrm{soc}}$, the module $V_{\mathrm{soc}}$ is the unique simple Gelfand-Tsetlin module having $\bar{v}+z$ in its support.

(v) For any parabolic subgroup $G \subset S_{\pi(\bar{v})}$, the quotient $\frac{\left|S_{\pi(\bar{v})}\right|}{|G|}$ appears as a Gelfand-Tsetlin multiplicity of the module $V_{\text {soc. }}$

In Section 6 we apply the above results to study the Gelfand-Tsetlin structure of Verma modules. Theorems 6.4 and 6.6 describe the support and the essential support of the simple singular Verma module $M(-\tilde{\rho})$, respectively, where $\tilde{\rho}=-(0,1, \ldots, n-1)$; notice that as $\mathfrak{s l}(n, \mathbb{C})$-module, this is isomorphic to the Verma module associated to minus the half-sum of the positive roots. Our second main result is summarized below.

Theorem 1.2. (i) Every Verma module is a submodule of a certain universal tableaux module $V(T(\bar{v}))$, and every simple Verma module appears as the socle of some $V(T(\bar{v}))$. In particular, $M(-\tilde{\rho})$ is the socle of $V(T(\mathbf{0}))$. 
(ii) The essential support of $M(-\tilde{\rho})$ is a rational cone. It contains weights with Gelfand-Tsetlin multiplicities $\frac{\prod_{i=1}^{n-1} i !}{|G|}$ for any standard parabolic subgroup $G$ of $S_{1} \times \ldots \times S_{n-1}$.

(iii) The maximal Gelfand-Tsetlin multiplicity in $M(-\tilde{\rho})$ is $\Pi_{i=1}^{n-1} i$.

The paper ends with a counterexample to the "hope" that the simple subquotients of $V(T(\bar{v}))$ have bases consisting of derivative tableaux. We show that this is not the case even for the simple Verma $\mathfrak{g l}(4, \mathbb{C})$-module $M(-\tilde{\rho})$.

Acknowledgements. We thank Ben Webster for pointing out that a conjecture in an earlier version of the paper was not true. V.F. is supported by CNPq grants (304467/2017-0 and 200783/2018-1). D.G. is supported in part by Simons Collaboration Grant 358245. L.E.R. is supported by Fapesp grant (2018/17955-7). P.Z. is supported by a CONICET postdoctoral fellowship.

\section{Preliminaries on the combinatorics of Gelfand-Tsetlin tableaux}

2.1. Notation. Given $a, b, k \in \mathbb{N}$ we set $\llbracket a, b \rrbracket=\{i \in \mathbb{N} \mid a \leq i \leq b\}$, with $\llbracket b \rrbracket=\llbracket 1, b \rrbracket$; also we denote by $S_{k}$ the symmetric group in $k$ elements. Given $\pi=\left(\pi_{1}, \ldots, \pi_{r}\right) \in \mathbb{N}^{r}$ with $\sum_{i} \pi_{i}=k$ we denote by $S_{\pi}$ the product $S_{\pi_{1}} \times S_{\pi_{2}} \times \cdots \times S_{\pi_{r}}$, which we see as a subgroup of $S_{k}$.

Fix $n \in \mathbb{N}$ and let $\mu=(1,2, \ldots, n)$. Given $\sigma \in S_{\mu}$ and $k \in \llbracket n \rrbracket$ we denote by $\sigma_{(k)}$ the projection of $\sigma$ to $S_{k}$. With a slight abuse of notation, we identify $S_{k}$ with the subgroup of $S_{\mu}$ consisting of elements $\sigma$ such that $\sigma_{(i)}$ is the identity for all $i \neq k$. Thus we can write $\sigma=\sigma_{(1)} \sigma_{(2)} \cdots \sigma_{(n)}$.

The group $S_{\mu}$ is a Coxeter group with generating set

$$
\left\{(i \mathrm{i}+1)_{(k)} \mid k \in \llbracket n \rrbracket, i \in \llbracket k-1 \rrbracket\right\},
$$

where $(i i+1)$ is the simple transposition interchanging $i$ and $i+1$. The usual notions of length, Bruhat order, parabolic subgroups, etc. will be considered with respect to this generating set. In particular, the length of $\sigma \in S_{\mu}$ is $\ell(\sigma)=\ell_{1}\left(\sigma_{(1)}\right)+\cdots+\ell_{n}\left(\sigma_{(n)}\right)$, where $\ell_{k}$ stands for the usual length in $S_{k}$. Also if $\tau \in S_{\mu}$ then $\sigma<\tau$ in the Bruhat order if and only if $\sigma_{(k)}<\tau_{(k)}$ for all $k$.

Henceforth we fix $\Sigma=\{(k, i) \mid 1 \leq i \leq k \leq n\}$. The group $S_{\mu}$ acts on $\Sigma$ with the action given by $\sigma \cdot(k, i)=\left(k, \sigma_{(k)}(i)\right)$. The subset $\Sigma^{\prime}=\{(k, i) \mid$ $1 \leq i \leq k \leq n-1\}$ is clearly invariant under this action.

For $k \geq a, b$ we set $\llbracket a, b \rrbracket_{k}=\{(k, i) \mid i \in \llbracket a, b \rrbracket\} \subset \Sigma$. Such a set will be called an interval of $\Sigma$, and given an interval $I=\llbracket a, b \rrbracket_{k}$ we write $a(I)=$ $a, b(I)=b, k(I)=k$. A partition of $\Sigma$ is a family of nonempty subsets of $\Sigma$, which we call blocks, whose disjoint union is $\Sigma$. An interval partition is 
a partition $\mathbb{I}$ whose blocks are intervals. We write $\mathbb{I}[k]$ for the set of all intervals $I \in \mathbb{I}$ with $k(I)=k$.

2.2. Let $\mathbb{C}^{\mu}=\mathbb{C} \times \mathbb{C}^{2} \times \cdots \times \mathbb{C}^{n}$, so every $v \in \mathbb{C}^{\mu}$ is an $n$-tuple $\left(v_{1}, v_{2}, \ldots, v_{n}\right)$ with $v_{k}=\left(v_{k, 1}, v_{k, 2}, \ldots, v_{k, k}\right) \in \mathbb{C}^{k}$. For each $(k, i) \in \Sigma$ we denote by $\delta^{k, i}$ the unique element in $\mathbb{C}^{\mu}$ such that $\left(\delta^{k, i}\right)_{l, j}=\delta_{k, l} \delta_{i, j}$, and refer to the set $\left\{\delta^{k, i} \mid(k, i) \in \Sigma\right\}$ as the canonical basis of $\mathbb{C}^{\mu}$. The group $S_{\mu}$ acts on $\mathbb{C}^{\mu}$ by linear operators whose action on the canonical basis is given by $\sigma \cdot \delta^{k, i}=\delta^{\sigma \cdot(k, i)}=\delta^{k, \sigma_{(k)}(i)}$. We denote by $\mathbb{Z}_{0}^{\mu}$ the additive subgroup of $\mathbb{C}^{\mu}$ generated by $\left\{\delta^{k, i} \mid(k, i) \in \Sigma^{\prime}\right\}$, which is stable under the action of $S_{\mu}$.

Given an interval $I=\llbracket a, b \rrbracket_{k}$ we will write $v(I)$ for $\left(v_{k, a}, v_{k, a+1}, \ldots, v_{k, b}\right)$. Given an interval partition $\mathbb{I}$ of $\Sigma$ we refer to the tuples $v(I)$ with $I \in \mathbb{I}$ as the blocks of $v$. We associate to $v$ a partition of $\Sigma$ denoted by $\mathbb{I}(v)$, where the block of $(n, i)$ is $\{(n, i)\}$, and for $k<n$ the block of $(k, i)$ is the set of all $(k, j)$ such that $v_{k, i}-v_{k, j} \in \mathbb{Z}$.

Definition 2.1. We say that $v \in \mathbb{C}^{\mu}$ is in normal form if whenever $v_{k, a}-v_{k, b} \in$ $\mathbb{Z}$ for some $a \leq b \leq k \leq n$, then $v_{k, i}-v_{k, j} \in \mathbb{Z}_{\geq 0}$ for all $a \leq i<j \leq b$.

Notice that if $v$ is in normal form then $\mathbb{I}(v)$ is an interval partition (but not the other way around). Clearly for each $v \in \mathbb{C}^{\mu}$ there exists at least one element in its $S_{\mu}$-orbit which is in normal form. Suppose $v$ is in normal form and let $\mathbb{I}(v)[k]=\left\{I_{1}, I_{2}, \ldots, I_{r}\right\}$, with $a\left(I_{i}\right)=b\left(I_{i-1}\right)+1$. We set $\pi(v, k)=\left(\left|I_{1}\right|,\left|I_{2}\right|, \ldots,\left|I_{r}\right|\right)$, so $S_{\pi(v, k)}$ is a parabolic subgroup of $S_{k}$; observe that by definition $S_{\pi(v, n)}$ is the trivial subgroup of $S_{n}$. We denote by $\pi(v)$ the concatenation of $\pi(v, 1), \ldots, \pi(v, n)$, and set $S_{\pi(v)}=$ $S_{\pi(v, 1)} \times S_{\pi(v, 2)} \times \cdots \times S_{\pi(v, n)} \subset S_{\mu}$. This is a parabolic subgroup of $S_{\mu}$.

2.3. The graph $\Omega(v)$. We now associate to each element $v \in \mathbb{C}^{\mu}$ a graph, which will be a major combinatorial tool in this paper. Given $v \in \mathbb{C}^{\mu}$ the graph $\Omega(v)$ is defined as follows: the set of vertices of $\Omega(v)$ is $\{[k, i]$ | $(k, i) \in \Sigma\}$, and we have an edge between $[k, i]$ and $[l, j]$ if and only if $v_{k, i}-v_{l, j} \in \mathbb{Z}$ and $|k-l| \leq 1$. We will use the notation $[k, i]-[l, j]$ for an edge between $[k, i]$ and $[l, j]$.

Definition 2.2. We say that $\bar{v} \in \mathbb{C}^{\mu}$ is a seed if it is in normal form and for $[k, i]$ and $[l, j]$ in the same connected component of $\Omega(v)$ the following holds: if $k, l<n$ then $\bar{v}_{k, i}=\bar{v}_{l, j}$, while if $l=n$ then $v_{k, i} \leq v_{n, j}$.

As mentioned before, $S_{\mu}$ acts on $\mathbb{C}^{\mu}$ and $\mathbb{Z}_{0}^{\mu}$ is stable under this action. Also, $\mathbb{Z}_{0}^{\mu}$ acts on $\mathbb{C}^{\mu}$ by translations: $z \cdot v=v+z$ for $z \in \mathbb{Z}_{0}^{\mu}$ and $v \in \mathbb{C}^{\mu}$. Thus the semidirect product $\mathbb{Z}_{0}^{\mu} \# S_{\mu}$ acts on $\mathbb{C}^{\mu}$. If $v$ is in normal form then there exists $z \in \mathbb{Z}_{0}^{\mu}$ such that $v+z$ is a seed. Hence for every $v \in \mathbb{C}^{\mu}$ there exists a seed in its $\left(\mathbb{Z}_{0}^{\mu} \# S_{\mu}\right)$-orbit. Two elements $v, w \in \mathbb{C}^{\mu}$ lie in the same orbit if and only if there exists $\sigma \in S_{\mu}$ such that $\sigma(\Omega(v))=\Omega(w)$ and 
$v-\sigma(w) \in \mathbb{Z}_{0}^{\mu}$, where $\sigma$ acts on the vertices of the graph in the obvious way.

Example 2.3. We will write elements of $\mathbb{C}^{\mu}$ as triangular arrays with $k$ entries in the $k$-th row counting from the bottom. In this example we assume that the set $\{1, a, b, c, \ldots\} \subset \mathbb{C}$ is linearly independent over $\mathbb{Z}$.

$$
\begin{array}{ccccc}
1 & a+1 & a & b & 0 \\
a & b-1 & b & a+1 \\
c & c+1 & c \\
a & a-1 \\
& a+1
\end{array}
$$

An element $v$ of $\mathbb{C}^{\mu}$

$$
\begin{aligned}
& \begin{array}{rrrrr}
a+1 & a & & 1 & 0 \\
a & & a & b-1 & b-1 \\
& & c & c & c
\end{array} \\
& a-1 a-1 \\
& a-1
\end{aligned}
$$

A seed in $\left(\mathbb{Z}_{0}^{\mu} \# S_{\mu}\right) v$

$$
\begin{array}{ccccc}
1 & 0 & a+1 & a & b \\
a+1 & a & b & b-1 \\
c+1 & c & c \\
4 & a-1 \\
& \\
4 &
\end{array}
$$

An element in normal form in $S_{\mu} v$

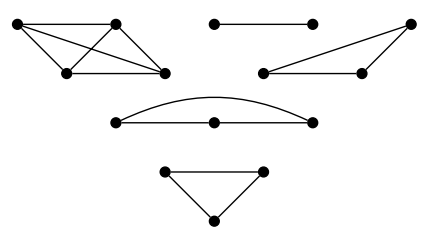

The graph of the previous elements

2.4. The set $\mathbb{D}(\bar{v})$. Recall that we denote by $\mathbb{Z}_{0}^{\mu}$ the set of all $z \in \mathbb{C}^{\mu}$ with $z_{k, i} \in \mathbb{Z}$ for all $(k, i) \in \Sigma$ and $z_{n, i}=0$ for all $i \in \llbracket n \rrbracket$.

Definition 2.4. Let $\bar{v} \in \mathbb{C}^{\mu}$ be a seed. We denote by $\mathbb{D}(\bar{v})$ the set of all $z \in \mathbb{Z}_{0}^{\mu}$ such that $z(I)$ is a nonincreasing sequence for all $I \in \mathbb{I}(\bar{v})$.

For the rest of this section we fix a seed $\bar{v}$ and set $\mathbb{I}=\mathbb{I}(\bar{v}), \pi=\pi(\bar{v})$ and $\mathbb{D}=\mathbb{D}(\bar{v})$. Notice that $\mathbb{D}$ is the set of those $z \in \mathbb{Z}_{0}^{\mu}$ such that $\bar{v}+z$ is in normal form. For example, if $\bar{v}$ is the zero vector $\mathbf{0} \in \mathbb{C}^{\mu}$ then $\mathbb{D}(\mathbf{0})$ is the set of $z \in \mathbb{Z}_{0}^{\mu}$ such that $z_{k, 1} \geq z_{k, 2} \geq \cdots \geq z_{k, k}$ for all $k \in \llbracket n-1 \rrbracket$.

Let $z \in \mathbb{D}$. The stabilizer of $z$ in $S_{\pi}$ is again a parabolic subgroup of $S_{\pi}$, which we denote as usual by $\left(S_{\pi}\right)_{z}$, so each coclass in $S_{\pi} /\left(S_{\pi}\right)_{z}$ has a unique minimal length representative. We denote by $S_{\pi}^{z}$ the set of these minimal length representatives, and refer to them as $z$-shuffles. Given $\sigma \in S_{\pi}$ we write $\sigma^{z}$ for the unique $z$-shuffle in $\sigma\left(S_{\pi}\right)_{z}$.

We denote by $\mathbb{I}(\bar{v}, z)$ the interval partition of $\Sigma$ where $(k, i)$ and $(k, j)$ lie in the same block if and only if $k<n$ and $(\bar{v}+z)_{k, i}=(\bar{v}+z)_{k, j}$. Equivalently, an interval lies in $\mathbb{I}(\bar{v}, z)$ if and only if it is an orbit of the action of $\left(S_{\pi}\right)_{z}$ on $\Sigma$. Let us say that $\sigma \in S_{\pi}$ is increasing, resp. decreasing, over an interval $\llbracket a, b \rrbracket_{k} \subset \Sigma$ if $\sigma_{(k)}(i)<\sigma_{(k)}(j)$, resp. $\sigma_{(k)}(i)>\sigma_{(k)}(j)$, whenever $a \leq i<j \leq b$. A permutation $\sigma$ is a $z$-shuffle if and only if it is increasing over every interval in $\mathbb{I}(\bar{v}, z)$, so $\sigma^{z}$ is the unique permutation 
in $S_{\pi}$ which is increasing over all intervals in $\mathbb{I}(\bar{v}, z)$ and such that $\sigma^{z}(z)=$ $\sigma(z)$.

Given an interval $I=\llbracket a, b \rrbracket_{k}$ we denote by $\omega(I)$ the permutation $i \mapsto$ $b+a-i$. This is the longest element in the symmetric group of the interval $I$, seen as a subgroup of $S_{\mu}$. It follows that the longest element in $\left(S_{\pi}\right)_{z}$ is $\prod_{I \in \mathbb{I}(\bar{v}, z)} \omega(I)$. We also write

$$
\begin{aligned}
& \alpha(I)=(b b-1 \ldots a)_{(k)}=(b b-1)_{(k)}(b-1 b-2)_{(k)} \cdots(a+1 a)_{(k)}, \\
& \beta(I)=(a a+1 \ldots b)_{(k)}=(a a+1)_{(k)}(a+1 a+2)_{(k)} \cdots(b-1 b)_{(k)} .
\end{aligned}
$$

These two permutations play a central role in the sequel. Notice that these permutations are mutual inverses.

Lemma 2.5. Let $z \in \mathbb{D}(\bar{v})$ and let $\omega_{0}$ be the longest element in $S_{\pi}$.

(a) We have $\omega_{0}^{z}=\omega_{0} \prod_{I \in \mathbb{I}(\bar{v}, z)} \omega(I)$.

(b) Given $(k, i) \in \Sigma$, we have $z+\delta^{k, i} \in \mathbb{D}(\bar{v})$ if and only if $i=a(I)$ for some $I \in \mathbb{I}(\bar{v}, z)[k]$, and $z-\delta^{k, i} \in \mathbb{D}(\bar{v})$ if and only if $i=b(I)$ for some $I \in \mathbb{I}(\bar{v}, z)[k]$.

(c) Let $I=\llbracket a, b \rrbracket_{k} \in \mathbb{I}(\bar{v}, z)$ and set $u=z+\delta^{k, a}, v=z-\delta^{k, b}$. There exist $\sigma, \tau \in S_{\pi}^{z}$ such that $\omega_{0}^{u}=\sigma \alpha(I)$ and $\omega_{0}^{v}=\tau \beta(I)$.

Proof. Put $\mathbb{I}^{\prime}=\mathbb{I}(\bar{v}, z)$. By definition $\omega_{0}$ is decreasing over each interval $I$ in $\mathbb{I}^{\prime}$. Since $\omega(I)$ is decreasing over $I$, it follows that $\omega_{0} \prod_{I \in \mathbb{I}^{\prime}} \omega(I)$ is increasing over every interval $I \in \mathbb{I}^{\prime}$, so it is a $z$-shuffle lying in the coclass $\omega_{0}\left(S_{\pi}\right)_{z}$. This proves part (a). Part (b) follows from the definitions.

To prove part (ㄷ) we only have to show that $\sigma=\omega_{0}^{u} \beta(I)$ and $\tau=\omega_{0}^{v} \alpha(I)$ are $z$-shuffles. We verify this for $\sigma$, as the verification for $\tau$ is similar. Let $J$ be the interval in $\mathbb{I}(\bar{v}, u)$ corresponding to $(k, a)$, so $J=\llbracket c, a \rrbracket_{k}$ for some $c \leq a$. Taking $J^{\prime}=J \backslash\{(k, a)\}$ and $I^{\prime}=I \backslash\{(k, a)\}$, by part (a),

$$
\begin{aligned}
\omega_{0}^{u} \beta(I) & =\omega_{0}\left(\prod_{K \in \mathbb{I}(\bar{v}, u), K \neq I^{\prime}, J} \omega(K)\right) \omega(J) \omega\left(I^{\prime}\right) \beta(I) \\
& =\omega_{0}\left(\prod_{K \in \mathbb{I}^{\prime}, K \neq I, J^{\prime}} \omega(K)\right) \omega(J) \omega\left(I^{\prime}\right) \beta(I) .
\end{aligned}
$$

It is enough to check that the composition of the product in the parenthesis with $\omega(J) \omega\left(I^{\prime}\right) \beta(I)$ is decreasing over the intervals of $\mathbb{I}^{\prime}$. This is immediate for $K \neq I, J^{\prime}$, so it remains to check that $\omega(J) \omega\left(I^{\prime}\right) \beta(I)$ is decreasing over $I, J^{\prime}$. This follows immediately from the definitions

\section{Background on universal tableaux Gelfand-Tsetlin modules}

Throughout this section we will work with the Lie algebra $\mathfrak{g l}(n, \mathbb{C})$. We denote by $\mathfrak{h}$ the Cartan subalgebra of diagonal matrices. We identify the dual of $\mathfrak{h}$ with $\mathbb{C}^{n}$ in the usual way. 
3.1. Generalities on Gelfand-Tsetlin modules. For each $k \in \llbracket n \rrbracket$ we denote by $U_{k}$ the universal enveloping algebra of $\mathfrak{g l}(k, \mathbb{C})$, and set $U=U_{n}$. By top-left-corner inclusion of matrices we obtain a chain

$$
\mathfrak{g l}(1, \mathbb{C}) \subset \mathfrak{g l}(2, \mathbb{C}) \subset \cdots \subset \mathfrak{g l}(n, \mathbb{C}),
$$

which in turn induces a chain $U_{1} \subset U_{2} \subset \cdots \subset U_{n}$. Denote by $Z_{k}$ the center of $U_{k}$ and by $\Gamma$ the subalgebra of $U$ generated by $\bigcup_{k=1}^{n} Z_{k}$. This is a maximal commutative subalgebra of $U$ called the Gelfand-Tsetlin subalgebra. It is generated by the elements

$$
c_{k, i}=\sum_{\left(r_{1}, \ldots, r_{i}\right) \in \llbracket k \rrbracket^{i}} E_{r_{1}, r_{2}} E_{r_{2}, r_{3}} \cdots E_{r_{i}, r_{1}} \quad(k, i) \in \Sigma .
$$

By a result of Zhelobenko, there exists an isomorphism

$$
\Gamma \rightarrow \mathbb{C}\left[x_{k, i} \mid(k, i) \in \Sigma\right]^{S_{\mu}}
$$

given by $c_{k, i} \mapsto \gamma_{k, i}$, where

$$
\gamma_{k, i}=\sum_{j=1}^{k}\left(x_{k, j}+k-1\right)^{i} \prod_{m \neq j}\left(1-\frac{1}{x_{k, j}-x_{k, m}}\right),
$$

see [FGR16, Subsection 3.1] for details. We will denote the image of $c \in \Gamma$ under this isomorphism by $\gamma_{c}$. It follows that $\operatorname{Specm} \Gamma \cong \mathbb{C}^{\mu} / S_{\mu}$, hence, every $v \in \mathbb{C}^{\mu}$ induces a character $\chi_{v}: \Gamma \rightarrow \mathbb{C}$ by setting $c \mapsto \gamma_{c}(v)$. Notice that $\chi_{v}=\chi_{w}$ if and only if $w$ lies in the $S_{\mu}$-orbit of $v$.

Definition 3.1. A U-module $M$ is called a Gelfand-Tsetlin module if it is finitely generated and

$$
M=\bigoplus_{\mathfrak{m} \in \operatorname{Specm} \Gamma} M[\mathfrak{m}],
$$

where $M[\mathfrak{m}]=\left\{x \in M \mid \mathfrak{m}^{k} x=0\right.$ for some $\left.k \geq 0\right\}$. The Gelfand-Tsetlin support, or simply the support, of $M$ is the set of all $\mathfrak{m}$ such that $M[\mathfrak{m}] \neq 0$, and will be denoted by supp $M$. For every $\mathfrak{m} \in \operatorname{Specm} \Gamma$ its Gelfand-Tsetlin multiplicity in $M$ is $\operatorname{dim} M[\mathfrak{m}]$.

Let $M$ be a Gelfand-Tsetlin module and let $v \in \mathbb{C}^{\mu}$. We put $M[v]=$ $M\left[\operatorname{ker} \chi_{v}\right]$, and denote by $p_{v}: M \rightarrow M[v]$ the projection map. We will identify the support of $M$ with the set of all $v \in \mathbb{C}^{\mu}$ such that $M[v] \neq 0$. We will say that the elements of $M[v]$ have Gelfand-Tsetlin weight $v$, and refer to $M[v]$ as the Gelfand-Tsetlin component of weight $v$. We will usually say "weight" instead of "Gelfand-Tsetlin weight". To avoid confusion we will sometimes use the expression "Cartan weight" for elements in the dual of $\mathfrak{h}$. Since $U(\mathfrak{h}) \subset \Gamma$ it follows that two elements with the same GelfandTsetlin weight have the same Cartan weight, but the converse does not hold. 
It is easy to check that a finitely-generated module $M$ is a GelfandTsetlin module if and only if for each $x \in M$ the complex vector space $\Gamma x$ has finite dimension. The following lemma is an immediate consequence of that observation.

Lemma 3.2. Let $M$ be a Gelfand-Tsetlin module and let $N \subset M$ be a U-submodule. Then $N$ is also a Gelfand-Tsetlin module. In particular for each $x \in N$ we have $p_{v}(x) \in N$ for all $v \in \mathbb{C}^{\mu}$.

3.2. Universal tableaux Gelfand-Tsetlin modules. Fix a seed $\bar{v}$ and set $\pi=\pi(\bar{v}), \mathbb{I}=\mathbb{I}(\bar{v})$ and $\mathbb{D}=\mathbb{D}(\bar{v})$. In [RZ18] a Gelfand-Tsetlin module $V(T(\bar{v}))$ is associated to any seed $\bar{v}$ (a similar construction appears in [EMV]). The module $V(T(\bar{v}))$ was called the "big Gelfand-Tsetlin module at $\bar{v}^{\prime \prime}$ in [RZ18], but here we refer to it as the universal tableaux module associated to $\bar{v}$. It is a module with $\mathbb{C}$-basis given by the set

$$
\left\{D_{\sigma}(\bar{v}+z) \mid z \in \mathbb{D}, \sigma \in S_{\pi}^{z}\right\}
$$

whose elements are called derivative tableaux. A tableau of the form $D_{e}(\bar{v}+$ $z$ ) is called the classical tableau associated to $\bar{v}+z$. Given $z \in \mathbb{D}(\bar{v})$ and $\sigma \in$ $S_{\pi}^{z}$ we denote by $D_{<\sigma}(\bar{v}+z)$ an arbitrary linear combination of tableaux $D_{\tau}(\bar{v}+z)$ with $\tau \in S_{\pi}^{z}$ strictly smaller than $\sigma$ in the induced Bruhat order (for details on the Bruhat order on shuffles see [BBo5, Section 2.5]).

We review the details regarding the explicit action of $U$ on $V(T(\bar{v}))$, which were proved in [FGRZ18]. Given $I=\llbracket a, b \rrbracket_{k}$ with $k<n$ we set

$$
e_{I}=\frac{\prod_{j=1}^{k+1}\left(x_{k, a}-x_{k+1, j}\right)}{\prod_{(k, j) \notin I}\left(x_{k, a}-x_{k, j}\right)} ; \quad f_{I}=\frac{\prod_{j=1}^{k-1}\left(x_{k, b}-x_{k-1, j}\right)}{\prod_{(k, j) \notin I}\left(x_{k, b}-x_{k, j}\right)} .
$$

Notice that if $I \in \mathbb{I}(\bar{v}, z)$ then $e_{I}(\bar{v}+z)$ and $f_{I}(\bar{v}+z)$ are well defined. We also set

$$
h_{k}=x_{k, 1}+\cdots+x_{k, k}-\left(x_{k-1,1}+\cdots+x_{k-1, k-1}\right)+k-1 .
$$

The following theorem is a direct consequence of [FGRZ18, Lemma 8.4].

Theorem 3.3. The action of the canonical generators of $\mathfrak{g l}(n, \mathbb{C})$ on $V(T(\bar{v}))$ is given by the formulas

$$
\begin{aligned}
E_{k, k+1} D_{\sigma}(\bar{v}+z) & =-\sum_{I \in \mathbb{I}(\bar{v}, z)[k]} \sum_{\tau \leq \sigma \alpha(I)} \mathfrak{D}_{\tau, \sigma \alpha(I)}^{\bar{v}+z}\left(e_{I}\right) D_{\tau}\left(\bar{v}+z+\delta^{k, a(I)}\right), \\
E_{k+1, k} D_{\sigma}(\bar{v}+z) & =\sum_{I \in \mathbb{I}(\bar{v}, z)[k]} \sum_{\tau \leq \sigma \beta(I)} \mathfrak{D}_{\tau, \sigma \beta(I)}^{\bar{v}+z}\left(f_{I}\right) D_{\tau}\left(\bar{v}+z-\delta^{k, b(I)}\right), \\
E_{k, k} D_{\sigma}(\bar{v}+z) & =h_{k}(\bar{v}+z) D_{\sigma}(\bar{v}+z)
\end{aligned}
$$


where $\mathfrak{D}_{\tau, \sigma}$ are the Postnikov-Stanley operators introduced in [FGRZ18, Definition 3.1], and elements $D_{\tau}(\bar{v}+u)$ such that $\tau$ is not a $u$-shuffle should be treated as zero.

Theorem 3.3 implies that every derivative tableau $D_{\sigma}(v)$ is a Cartan weight vector of weight $\lambda=\left(h_{1}(v), \cdots, h_{n}(v)\right)$. Hence $V(T(\bar{v}))$ is a (Car$\tan )$ weight representation with infinite-dimensional weight spaces.

Remark 3.4. We record here for future reference that $\mathfrak{D}_{\tau, \sigma}$ is a differential operator of degree $\ell(\sigma)-\ell(\tau)$. In particular $\mathfrak{D}_{\sigma, \sigma}^{v}$ is the evaluation at $v$, which allows to rewrite the formulas in a simplified form as

$$
\begin{gathered}
E_{k, k+1} D_{\sigma}(\bar{v}+z)=-\sum_{I \in \mathbb{I}(\bar{v}, z)[k]}\left(e_{I}(\bar{v}+z) D_{\sigma \alpha(I)}\left(\bar{v}+z+\delta^{k, a(I)}\right)\right. \\
\left.+D_{<\sigma \alpha(I)}\left(\bar{v}+z+\delta^{k, a(I)}\right)\right), \\
E_{k+1, k} D_{\sigma}(\bar{v}+z)=\sum_{I \in \mathbb{I}(\bar{v}, z)[k]}\left(f_{I}(\bar{v}+z) D_{\sigma \beta(I)}\left(\bar{v}+z-\delta^{k, b(I)}\right)\right. \\
\left.+D_{<\sigma \beta(I)}\left(\bar{v}+z-\delta^{k, b(I)}\right)\right),
\end{gathered}
$$

though we must keep in mind that in some cases $D_{\sigma \alpha(I)}\left(\bar{v}+z+\delta^{k, a(I)}\right)$ and $D_{\sigma \beta(I)}\left(\bar{v}+z-\delta^{k, b(I)}\right)$ are zero. This happens when $z_{k, a(I)}+1=z_{k, a(I)-1}$ and $z_{k, b(I)}-1=z_{k, b(I)+1}$ respectively.

The following proposition shows that $V(T(\bar{v}))$ is a Gelfand-Tsetlin module and describes the Gelfand-Tsetlin weight components of $V(T(\bar{v}))$. For the proofs of the statements see [FGRZ18, Proposition 6.4 and Lemma 6.5].

Proposition 3.5. Let $z \in \mathbb{D}$, let $\mathfrak{m}=\operatorname{ker} \chi_{\bar{v}+z}$ and let $T=\sum_{\sigma} a_{\sigma} D_{\sigma}(\bar{v}+z)$.

(a) If $c \in \Gamma$ then

$$
\begin{aligned}
c D_{\sigma}(\bar{v}+z) & =\gamma_{c}(\bar{v}+z) D_{\sigma}(\bar{v}+z)+\sum_{\tau<\sigma} \mathfrak{D}_{\tau, \sigma}^{\bar{v}+z}\left(\gamma_{c}\right) D_{\tau}(\bar{v}+z) \\
& =\gamma_{c}(\bar{v}+z) D_{\sigma}(\bar{v}+z)+D_{<\sigma}(\bar{v}+z) .
\end{aligned}
$$

(b) The Gelfand-Tsetlin component $V(T(\bar{v}))[\mathfrak{m}]$ is cyclic as a $\Gamma$-module, and $T$ is a cyclic vector if and only if $a_{\omega_{0}^{z}} \neq 0$.

(c) Let $r>0$. Then $\mathfrak{m}^{r} T=0$ if and only if $a_{\sigma}=0$ for all $\ell(\sigma) \geq r$. In particular, $\mathfrak{m}^{\ell\left(\omega_{0}^{z}\right)+1} V(T(\bar{v}))[\mathfrak{m}]=0$, and the only simultaneous $\Gamma$-eigenvector of eigenvalue $\chi_{\bar{v}+z}$, up to a scalar multiple, is $D_{e}(\bar{v}+z)$.

(d) Let $r=\ell\left(\omega_{0}^{z}\right)$. Given $c \in \Gamma$ let $c_{\bar{v}+z}$ be the restriction of $c$ on $V(T(\bar{v}))[\mathfrak{m}]$. Then the set of all $c$ such that the Jordan form of $c_{\bar{v}+z}$ has exactly one block of size $r$ projects to a Zariski open set in $\Gamma / \mathfrak{m}^{r+1}$. If $c_{\bar{v}+z}$ falls outside this set then its Jordan form contains blocks only of size strictly smaller than $r$. 
It follows from this proposition that $V(T(\bar{v}))[\bar{v}+z]$ is the $\mathbb{C}$-span of the derivative tableaux $D_{\sigma}(\bar{v}+z)$. Notice that, through the map $D_{\sigma}(\bar{v}+$ $z) \mapsto \bar{v}+\sigma(z)$, the support of $V(T(\bar{v}))$ can be identified with $\bar{v}+\mathbb{Z}_{0}^{\mu}$, and the Gelfand-Tsetlin multiplicity of $\bar{v}+z$ is precisely the cardinality of its $S_{\pi}$-orbit. In [FO14, Theorem 4.12 (c)] Futorny and Ovsienko proved that this is a bound for the dimension of the Gelfand-Tsetlin component of weight $\bar{v}+z$ of a simple Gelfand-Tsetlin module and conjectured that the bound is sharp. In particular these dimensions are bounded by $(n-$ $1) !(n-2) ! \cdots 2$ !. As explained in the introduction, the conjecture can be proved using results from [FGRZ18] or [EMV]. We will refine this result in the following sections.

\section{Cyclic submodules of universal tableaux modules}

Again, we fix a seed $\bar{v}$ and set $\pi=\pi(\bar{v}), \mathbb{I}=\mathbb{I}(\bar{v}), \mathbb{D}=\mathbb{D}(\bar{v})$.

4.1. The oriented graph of $\bar{v}+z$. Recall that $\Omega(v)$ is the graph with vertex set $\Sigma$ and edges $[k, i]-[l, j]$ whenever $v_{k, i}-v_{l, j} \in \mathbb{Z}$ and $|k-l| \leq 1$; in particular $\Omega(\bar{v})=\Omega(\bar{v}+z)$ for all $z \in \mathbb{Z}_{0}^{\mu}$. In what follows we will define for each $z \in \mathbb{D}$ an orientation of the graph $\Omega(\bar{v}+z)$ and denote by $\vec{\Omega}(\bar{v}+$ $z)$ the resulting oriented graph. As usual, we will use the notation $[k, i] \rightarrow$ $[l, j]$ for "the oriented edge with tail $[k, i]$ and head $[l, j]$ ".

Definition 4.1. Let $z \in \mathbb{D}$. The oriented graph $\vec{\Omega}(\bar{v}+z)$ has $\Omega(\bar{v}+z)$ as its underlying graph, and its orientation is subject to the following three rules.

(i) If $[k, i]-[k, j]$ is an edge with $i<j$, then $[k, i] \rightarrow[k, j]$ is an edge of $\vec{\Omega}(\bar{v}+z)$.

(ii) If $[k, i]-[k-1, j]$ is an edge such that $(\bar{v}+z)_{k, i}-(\bar{v}+z)_{k-1, j} \in \mathbb{Z}_{\geq 0}$, then $[k, i] \rightarrow[k-1, j]$ is an edge of $\vec{\Omega}(\bar{v}+z)$.

(iii) If $[k, i]-[k-1, j]$ is an edge such that $(\bar{v}+z)_{k, i}-(\bar{v}+z)_{k-1, j} \in \mathbb{Z}_{<0}$, then $[k-1, j] \rightarrow[k, i]$ is an edge of $\vec{\Omega}(\bar{v}+z)$.

We denote by $\Omega^{+}(\bar{v}+z)$ the subgraph of $\Omega(\bar{v}+z)$ obtained by keeping only the edges of the form $[k, i] \rightarrow[k-1, j]$ in $\vec{\Omega}(\bar{v}+z)$. Analogously we denote by $\Omega^{-}(\bar{v}+z)$ the subgraph obtained by keeping only the edges of the form $[k-$ $1, j] \rightarrow[k, i]$ in $\vec{\Omega}(\bar{v}+z)$.

Notice that $\Omega^{+}(\bar{v}+z)$ is an unoriented graph (though by its definition it is easy to recover the orientation of its edges in $\vec{\Omega}(\bar{v}+z))$. Note also that the above definition can be considered as a refined version of the graph associated to a set of relations introduced in $\S 4$ of [FRZ19]. In the latter case, arrows between vertices on the $k$-th row are not allowed for $k<n$.

It follows from the definition that $\vec{\Omega}(\bar{v}+z)$ has no loops, so each of its connected components has at least one source (a vertex that is not the 
head of any edge) and at least one sink (a vertex that is not the tail of any edge). It also follows that $\Omega^{-}(\bar{v})$ is the graph with vertex set $\Sigma$ and no edges, while for every $z \in \mathbb{D}$ any edge of $\Omega^{+}(\bar{v})$ is an edge of either $\Omega^{+}(\bar{v}+z)$ or of $\Omega^{-}(\bar{v}+z)$.

Fix $z \in \mathbb{D}$ and let $\vec{\Omega}=\vec{\Omega}(\bar{v}+z)$. We next introduce a reduced version of the graph $\vec{\Omega}$ from which it can be recovered. We say that a directed edge $[k, i] \rightarrow[l, j]$ in $\vec{\Omega}$ is superfluous if there exists a path of directed edges $[k, i]=\left[k_{0}, i_{0}\right] \rightarrow\left[k_{1}, i_{1}\right] \rightarrow \cdots \rightarrow\left[k_{r}, i_{r}\right]=[l, j]$ with $r>1$. The reduced graph of $\bar{v}+z$, denoted by $\widetilde{\Omega}(\bar{v}+z)$, is the oriented subgraph obtained by removing all superfluous edges.

Since $\vec{\Omega}(\bar{v}+z)$ is a directed graph without loops, so is $\widetilde{\Omega}(\bar{v}+z)$. We recover $\vec{\Omega}(\bar{v}+z)$ from $\widetilde{\Omega}(\bar{v}+z)$ by adding a directed edge $[k, i] \rightarrow[l, j]$ whenever there is a path from $[k, i]$ to $[l, j]$ in $\widetilde{\Omega}(\bar{v}+z)$ and $|k-l| \leq 1$. Thus given $y \in \mathbb{D}$ we have that $\vec{\Omega}(\bar{v}+z)=\vec{\Omega}(\bar{v}+y)$ if and only if $\widetilde{\Omega}(\bar{v}+z)=\widetilde{\Omega}(\bar{v}+y)$.

Example 4.2. Below we show a few examples of reduced graphs $\widetilde{\Omega}(\bar{v}+z)$.

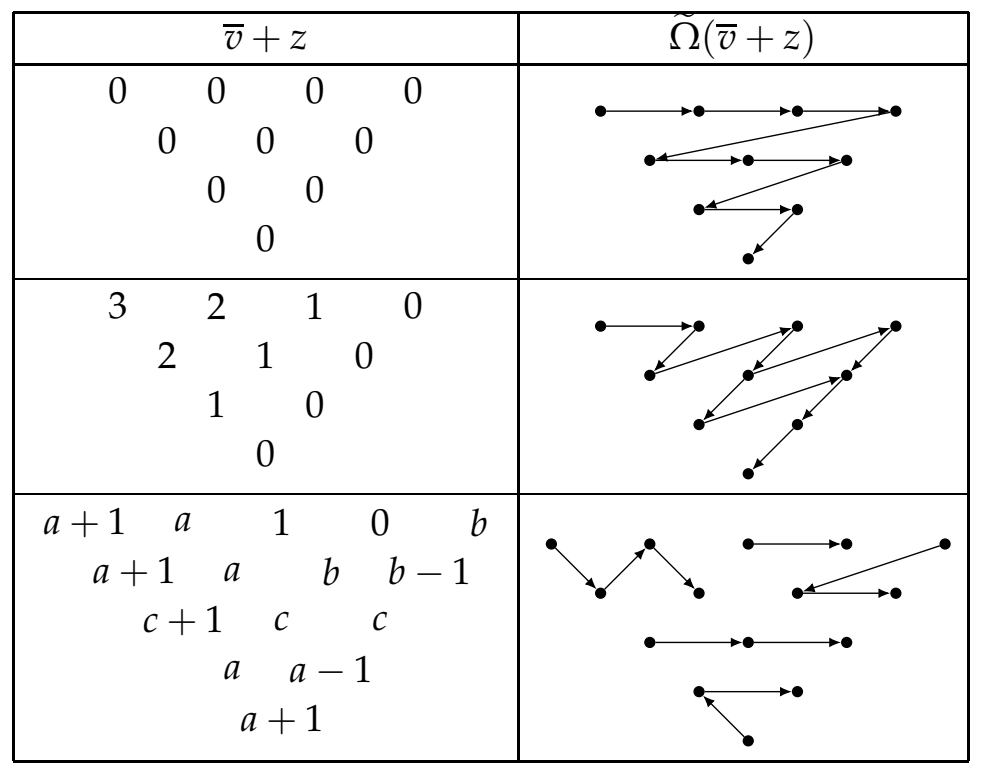

4.2. Cyclic submodules. We now begin with our study of the internal structure of $V(T(\bar{v}))$. We use the notation $\Omega^{ \pm}(x) \subset \Omega^{ \pm}(y)$ to indicate that the edge set of $\Omega^{ \pm}(x)$ is contained in that of $\Omega^{ \pm}(y)$. We also denote by $\varnothing$ the graph with vertex set $\Sigma$ and no edges. The following lemma is a generalization of [FGR15. Theorem 6.8].

Lemma 4.3. Let $y, z \in \mathbb{D}$. If $\Omega^{+}(\bar{v}+z) \subset \Omega^{+}(\bar{v}+y)$, or equivalently if $\Omega^{-}(\bar{v}+y) \subset \Omega^{-}(\bar{v}+z)$, then the following hold. 
(a) $D_{e}(\bar{v}+y) \in U D_{e}(\bar{v}+z)$.

(b) $D_{\omega_{0}^{y}}(\bar{v}+y) \in U D_{\omega_{0}^{z}}(\bar{v}+z)$.

Proof. We apply induction on $|y-z|=\sum_{(k, i) \in \Sigma}\left|y_{k, i}-z_{k, i}\right|$. That is, we will show that it is possible to choose $(k, i) \in \Sigma$ and $u=z \pm \delta^{k, i}$ with the sign chosen so that $|y-u|<|y-z|$ and the following hold:

(1) $u \in \mathbb{D}$,

(2) $\Omega^{+}(\bar{v}+z) \subset \Omega^{+}(\bar{v}+u) \subset \Omega^{+}(\bar{v}+y)$,

(3) $D_{e}(\bar{v}+u) \in U D_{e}(\bar{v}+z)$, and

(4) $D_{\omega_{0}^{u}}(\bar{v}+u) \in U D_{\omega_{0}^{z}}(\bar{v}+z)$.

Clearly the lemma follows from the existence of such $u$.

Denote by $\Omega_{<}$, respectively $\Omega_{>}$, the induced subgraph of $\vec{\Omega}(\bar{v}+y)$ with vertex set consisting of those $[k, i]$ such that $z_{k, i}<y_{k, i}$, respectively $z_{k, i}>y_{k, i}$; here induced means that there is a directed edge between two vertices of the subgraph if and only if there was a directed edge between these vertices in the original graph. Notice that no vertex of the form $[n, i]$ is in either graph. If both $\Omega_{<}$and $\Omega_{>}$are empty then $y=z$ and there is nothing to prove. Suppose $\Omega_{<}$is not empty. Then, since it is an oriented subgraph of $\vec{\Omega}(\bar{v}+y)$, it has no loops and hence has at least one source, say $[k, i]$. We claim that (1), (2), (3) and (4) hold with $u=z+\delta^{k, i}$. If $\Omega_{<}$ is empty then we take $[k, i]$ to be a sink in $\Omega_{>}$and set $u=z-\delta^{k, i}$. We now proceed with the proof assuming $\Omega_{<}$is not empty. The other case is similar and we discuss how to adapt the proof at each step.

Proof of (1). By Lemma 2.5(b), it is enough to show that if $[k, i-1] \rightarrow[k, i]$ is an edge of $\vec{\Omega}(\bar{v})$ then $z_{k, i-1}>z_{k, i}$. If this edge is indeed present then since $y \in \mathbb{D}$ we know that $y_{k, i-1} \geq y_{k, i}$. On the other hand since $[k, i]$ is a source of $\Omega_{<}$we must have $z_{k, i-1} \geq y_{k, i-1}$ and $z_{k, i}<y_{k, i}$, and these inequalities imply the one we are looking for.

Proof of (2). To show that $\Omega^{+}(\bar{v}+z) \subset \Omega^{+}(\bar{v}+u)$ it is enough to prove that if either $[k, i]-[k-1, j]$ or $[k+1, j]-[k, i]$ is an edge of $\Omega^{+}(\bar{v}+z)$ then it is also an edge of $\Omega^{+}(\bar{v}+u)$. The first case is obvious. For the second, the choice of $(k, i)$ as a source of $\Omega_{<}$implies that $z_{k+1, j}=u_{k+1, j} \geq y_{k+1, j}$ while $u_{k, i}=z_{k, i}+1 \leq y_{k, i}$. Since $\Omega^{+}(\bar{v}+z) \subset \Omega^{+}(\bar{v}+y)$ we see that $u_{k+1, j} \geq y_{k+1, j} \geq y_{k, i} \geq u_{k, i}$, so the edge $[k+1, j]-[k, i]$ is in $\Omega^{+}(\bar{v}+u)$.

To show that $\Omega^{+}(\bar{v}+u) \subset \Omega^{+}(\bar{v}+y)$ we again need to consider only edges of the form $[k+1, j]-[k, i]$ and $[k, i]-[k-1, j]$ of the first graph. In the first case we have $z_{k+1, j}=u_{k+1, j} \geq u_{k, i}=z_{k, i}+1$, so $[k+1, j]-[k, i]$ is an edge of $\Omega^{+}(\bar{v}+z)$, and by the hypothesis it is also and edge of $\Omega^{+}(\bar{v}+y)$. In the second case we have $z_{k, i}+1=u_{k, i} \geq u_{k-1, j}=z_{k-1, j}$. If the inequality is strict then $[k, i]-[k-1, j]$ is an edge of $\Omega^{+}(\bar{v}+z)$, and hence of $\Omega^{+}(\bar{v}+y)$. If on the other hand equality holds, then $z_{k, i}<z_{k-1, j}$ and so $[k-1, j] \rightarrow[k, i]$ is an edge of $\vec{\Omega}(\bar{v}+z)$. Since $[k, i]$ is a source 
of $\Omega_{<,}$, we must have that $y_{k-1, j} \leq z_{k-1, j}=z_{k, i}+1 \leq y_{k, i}$, and hence $[k, i]-[k-1, j]$ is an edge of $\Omega^{+}(\bar{v}+y)$.

Proof of (3). Let $I \in \mathbb{I}(\bar{v}, z)$ be the interval containing $(k, i)$. It follows from the definitions that $\alpha(I)$ is a $u$-shuffle. Using the formulas for the action of $U$ as given in Remark 3.4, we see that

$$
p_{\bar{v}+u}\left(E_{k, k+1} D_{e}(\bar{v}+z)\right)=e_{I}(\bar{v}+z) D_{\alpha(I)}(\bar{v}+u)+D_{<\alpha(I)}(\bar{v}+u),
$$

where $p_{v}$ is the projection to the Gelfand-Tsetlin component $V(T(\bar{v}))[v]$. By Lemma 3.2, this element lies in $U D_{e}(\bar{v}+z)$.

If $e_{I}(\bar{v}+z)=0$, then there is some $(k+1, j) \in \Sigma$ such that $z_{k+1, j}=z_{k, i}$, which implies that $[k+1, j]-[k, i]$ is an edge of $\Omega^{+}(\bar{v}+z)$ and, hence, $y_{k+1, j} \geq y_{k, i}$. On the other hand since $[k, i]$ is a source of $\Omega_{<}$we have $z_{k, i}<y_{k, i} \leq y_{k+1, j} \leq z_{k+1, j}$, which leads to a contradiction. Thus we see that $p_{\bar{v}+u}\left(E_{k, k+1} D_{e}(\bar{v}+z)\right) \neq 0$. By Proposition $3 \cdot 5($ (C) $)$ this implies that $D_{e}(\bar{v}+u) \in U D_{e}(\bar{v}+z)$. The proof in the second case is analogous, except that we must look at $p_{\bar{v}+u}\left(E_{k+1, k} D_{e}(\bar{v}+z)\right)$, and instead of $e_{I}(\bar{v}+z) \neq 0$, we show that $f_{I}(\bar{v}+z) \neq 0$.

Proof of (4). Recall from Lemma $2.5(\mathrm{C})$ that there exists $\sigma \in S_{\pi}^{z}$ such that $\omega_{0}^{u}=\sigma \alpha(I)$. By Proposition $3 \cdot 5(\underline{b}), D_{\sigma}(\bar{v}+z) \in U D_{\omega_{0}^{z}}(\bar{v}+z)$. Again by the formulas in Remark 3.4 and by Lemma 3.2 .

$$
\begin{aligned}
& p_{\bar{v}+u}\left(E_{k, k+1} D_{\sigma}(\bar{v}+z)\right)= \\
& \quad e_{I}(\bar{v}+z) D_{\sigma \alpha(I)}(\bar{v}+u)+D_{<\alpha(I)}(\bar{v}+u) \in U D_{\omega_{0}^{z}}(\bar{v}+z) .
\end{aligned}
$$

As we saw in the proof of the previous point, the leading coefficient is nonzero. By Proposition $3 \cdot 5(\sqrt[b]{b})$ this element generates the GelfandTsetlin weight component $V(T(\bar{v}))[\bar{v}+u]$, so in particular $D_{\omega_{0}^{u}}(\bar{v}+u) \in$ $U D_{\omega_{0}^{z}}(\bar{v}+z)$. For the second case we must take $p_{\bar{v}+u}\left(E_{k, k+1} D_{\tau}(\bar{v}+z)\right)$, with $\tau$ as in Lemma $2.5($ C $)$, and then the rest of the proof is similar.

4.3. The socle of $V(T(\bar{v}))$. An element $z \in \mathbb{D}$ is said to be fully critical if $z_{k, i}=z_{k, j}$ whenever $[k, i]-[k, j]$ is an edge of $\Omega(\bar{v}+z)$. Equivalently, $z$ is fully critical if and only if $\left(S_{\pi}\right)_{z}=S_{\pi}$. The following proposition shows that $V(T(\bar{v}))$ is a cyclic module, and that it has a unique minimal module. Item $\mathrm{C}$ of the next Proposition is a special case of [EMV, Theorem 11] and [FGRZ18, Theorem 8.5]. We include it here for completeness.

Proposition 4.4. Let $z \in \mathbb{D}$ be fully critical.

(a) If $\Omega^{+}(\bar{v}+z)=\varnothing$ then $U D_{e}(\bar{v}+z)=V(T(\bar{v}))$. In particular $V(T(\bar{v}))$ is a cyclic module.

(b) If $\Omega^{-}(\bar{v}+z)=\varnothing$ then $U D_{e}(\bar{v}+z)$ is simple and contained in any other submodule of $V(T(\bar{v}))$.

(c) If $\Omega(\bar{v})$ has no edges of the form $[k, i]-[k-1, j]$ then $V(T(\bar{v}))$ is simple.

Proof. Notice that it is always possible to find fully critical elements $z \in \mathbb{D}$ satisfying the hypothesis of the first item. If $z$ is such an element, being 
fully critical, then $\omega_{0}^{z}=e$. Also by Lemma $4 \cdot 3(\mathbb{b})$, every derivative tableau $D_{\omega_{0}^{y}}(\bar{v}+y)$ with $y \in \mathbb{D}$ is in $U D_{e}(\bar{v}+z)$. Thus

$$
U D_{e}(\bar{v}+z)[\bar{v}+y] \supset \Gamma D_{\omega_{0}^{y}}(\bar{v}+y)=V(T(\bar{v}))[\bar{v}+y]
$$

and this proves part (a).

Assume now that $\Omega^{-}(\bar{v}+z)=\varnothing$, and set $N=U D_{e}(\bar{v}+z)$. Then for any $y \in \mathbb{D}$ we have $\Omega^{+}(\bar{v}+y) \subset \Omega^{+}(\bar{v}+z)$, so by Lemma 4.3(1.a) $N \subset$ $U D_{e}(\bar{v}+y)$. Since every submodule of $V(T(\bar{v}))$ contains some tableaux of this form, it follows that $N$ is contained in every submodule of $V(T(\bar{v}))$ which proves part (b). Part (ㄷ) is an easy consequence of (a) and (b).

As mentioned in the proof of the Proposition, if $\Omega^{-}(\bar{v}+z)=\varnothing$ then $U D_{e}(\bar{v}+z)$ is minimal among the submodules of $V(T(\bar{v}))$.

Corollary 4.5. The socle of $V(T(\bar{v}))$ is simple and equal to $U D_{e}(\bar{v}+z)$ for any $z$ such that $\Omega^{-}(\bar{v}+z)=\varnothing$. In particular, $\operatorname{soc} V(T(\bar{v}))=U D_{e}(\bar{v})$.

\section{The essential support of the socle and a proof of the Strong Futorny-Ovsienko conjecture}

As before, $\bar{v}$ is a fixed seed and $\pi=\pi(\bar{v}), \mathbb{I}=\mathbb{I}(\bar{v}), \mathbb{D}=\mathbb{D}(\bar{v})$.

5.1. The essential support of the socle of $V(T(\bar{v}))$. We denote the socle of $V(T(\bar{v}))$ by $V_{\text {soc }}$. As mentioned above $V_{\text {soc }}$ is simple. The dimension of the Gelfand-Tsetlin component $V_{\mathrm{soc}}[\bar{v}+z]$ is bounded by $\operatorname{dim} V[\bar{v}+z]=\left|S_{\pi}^{z}\right|$ (this is the same bound given in [FO14, Theorem 4.12 (c)]).

Definition 5.1. Let $z \in \mathbb{D}$ and let $M$ be a simple Gelfand-Tsetlin module such that $M[\bar{v}+z] \neq 0$. We say that $\bar{v}+z$ is in the essential support of $M$ if $\operatorname{dim} M[\bar{v}+z]=\left|S_{\pi}^{z}\right|$. We denote the essential support of $M$ by essupp $M$.

We introduce the following notations

$$
\begin{aligned}
\operatorname{supp}(\bar{v}) & =\left\{z \in \mathbb{D} \mid \operatorname{dim} V_{\text {soc }}[\bar{v}+z] \neq 0\right\} \\
\operatorname{ess}(\bar{v}) & =\left\{z \in \mathbb{D} \mid \bar{v}+z \in \operatorname{essupp} V_{\text {soc }}\right\}
\end{aligned}
$$

In view of the fact that $V_{\text {soc }}$ is simple, its essential support is the set of those $z \in \mathbb{D}$ for which the dimension of $V_{\mathrm{soc}}[\bar{v}+z]$ is as large as the FutornyOvsienko bound allows.

5.2. Cones associated to the support. Denote by $\mathbb{R}^{\mu}$ the set of points in $\mathbb{C}^{\mu}$ with real coordinates. Recall that a rational polyhedral cone is the intersection of finitely many half-spaces $\left\{x \in \mathbb{R}^{\mu} \mid \varphi(x) \geq q\right\}$ where $\varphi$ is a linear functional with rational coefficients in the canonical basis and $q \in \mathbb{Q}$. The rank of a cone is the dimension of the smallest affine space that contains it. 
Set

$$
\begin{aligned}
& \mathcal{S}=\left\{\Omega^{+}(\bar{v}+z) \mid z \in \operatorname{supp}(\bar{v})\right\} \\
& \mathcal{E}=\left\{\Omega^{+}(\bar{v}+z) \mid z \in \operatorname{ess}(\bar{v})\right\} .
\end{aligned}
$$

These are finite sets, which we order by the relation of being a subgraph. For each $\Omega \subset \Omega^{+}(\bar{v})$ we set

$$
\begin{aligned}
& \mathcal{P}(\Omega)=\left\{z \in \mathbb{D} \mid \Omega=\Omega^{+}(\bar{v}+z)\right\} ; \\
& \overline{\mathcal{P}}(\Omega)=\left\{z \in \mathbb{D} \mid \Omega \subset \Omega^{+}(\bar{v}+z)\right\} .
\end{aligned}
$$

By Lemma $4 \cdot 3$ if $z \in \operatorname{supp}(\bar{v})$ then $\mathcal{P}\left(\Omega^{+}(\bar{v}+z)\right) \subset \overline{\mathcal{P}}\left(\Omega^{+}(\bar{v}+z)\right) \subset$ $\operatorname{supp}(\bar{v})$, and the same holds if we replace $\operatorname{supp}(\bar{v})$ with ess $(\bar{v})$. Denoting by $\mathcal{S}_{0}$ and $\mathcal{E}_{0}$ the set of minimal elements of $\mathcal{S}$ and $\mathcal{E}$ respectively we see that

$$
\begin{aligned}
\operatorname{supp}(\bar{v}) & =\bigcup_{\Omega \in \mathcal{S}} \mathcal{P}(\Omega)=\bigcup_{\Omega \in \mathcal{S}_{0}} \overline{\mathcal{P}}(\Omega) ; \\
\operatorname{ess}(\bar{v}) & =\bigcup_{\Omega \in \mathcal{E}} \mathcal{P}(\Omega)=\bigcup_{\Omega \in \mathcal{E}_{0}} \overline{\mathcal{P}}(\Omega) .
\end{aligned}
$$

Let $w \in \mathbb{D}$ and $z \in \mathbb{Z}_{0}^{\mu}$, and put $\vec{\Omega}=\vec{\Omega}(\bar{v}+w)$. We have that $z \in$ $\mathcal{P}\left(\Omega^{+}(\bar{v}+w)\right)$ if and only if it satisfies the following conditions for all $k<n$ :

(a) if $[n, i] \rightarrow[n-1, j]$ is a directed edge of $\vec{\Omega}$ then $z_{n-1, j} \leq \bar{v}_{n, i}-\bar{v}_{n-1, j}$;

(b) if $[n-1, j] \rightarrow[n, i]$ is a directed edge of $\vec{\Omega}$ then $z_{n-1, j}>\bar{v}_{n, i}-\bar{v}_{n-1, j}$;

(c) if $[k, i] \rightarrow[k-1, j]$ is a directed edge of $\vec{\Omega}$ then $z_{k-1, j} \leq z_{k, j}$;

(d) if $[k-1, i] \rightarrow[k, j]$ is a directed edge of $\vec{\Omega}$ then $z_{k-1, j}>z_{k, j}$;

(e) if $[k, i] \rightarrow[k, i+1]$ is a directed edge of $\vec{\Omega}$ then $z_{k, i} \geq z_{k, i+1}$.

Furthermore, $z \in \overline{\mathcal{P}}\left(\Omega^{+}(\bar{v}+w)\right)$ if and only if it satisfies conditions $(a),(c)$ and $(e)$ (this last condition guarantees that $z \in \mathbb{D}$ ). It follows that both $\mathcal{P}\left(\Omega^{+}(\bar{v}+w)\right)$ and $\overline{\mathcal{P}}\left(\Omega^{+}(\bar{v}+w)\right)$ are the set of integral points of a rational polyhedral cone. Since these sets are contained in $\mathbb{Z}_{0}^{\mu}$ their rank is at most $\frac{n(n-1)}{2}$.

Theorem 5.2. Both the support and the essential support of $V_{\mathrm{soc}}$ are the set of integral points of a finite union of polyhedral rational cones. Furthermore, one of these cones has rank $\frac{n(n-1)}{2}$.

Proof. We have already shown that both supp $V_{\text {soc }}$ and essupp $V_{\text {soc }}$ can be written as a union of sets of the form $\overline{\mathcal{P}}(\Omega)$ for appropriate subgraphs of $\Omega(\bar{v})$, so the first part of the theorem is proved.

For the second part, we will show show that $\mathcal{P}\left(\Omega^{+}(\bar{v})\right)=\overline{\mathcal{P}}\left(\Omega^{+}(\bar{v})\right)$ is the set of integral points of a cone of rank $\frac{n(n-1)}{2}$. Given $1 \leq i \leq k \leq n-1$ 
let $c^{k, i} \in \mathbb{Z}_{0}^{\mu}$ be such that $c_{l, j}^{k, i}$ is -1 if there is a directed path in $\vec{\Omega}(\bar{v})$ from $[k, i]$ to $[l, j]$, and 0 otherwise. Here we assume that $[k, i]$ is linked to itself by a trivial path, so $c_{k, i}^{k, i}=-1$. Then $\Omega^{+}\left(\bar{v}+c^{k, i}\right)=\Omega^{+}(\bar{v})$ and so $c^{k, i} \in \mathcal{P}\left(\Omega^{+}(\bar{v})\right)$. Now if $z$ is any nonzero element in $\mathcal{P}\left(\Omega^{+}(\bar{v})\right)$ and $z_{k, i} \neq 0$ then $\Omega^{+}\left(\bar{v}+z-c^{k, i}\right)=\Omega^{+}(\bar{v})$. By induction $\mathcal{P}\left(\Omega^{+}(\bar{v})\right)$ is the monoid generated by the $c^{k, i}$, and hence has the desired rank.

Now we are ready to prove the Strong Futorny-Ovsienko Conjecture. For each standard parabolic subgroup $G \subset S_{\pi}$ there exists $z$ in $\mathcal{P}(\Omega(\bar{v}))$ such that $G=\left(S_{\pi}\right)_{z}$. Indeed, the orbits of $G$ form an interval partition of $\Sigma^{\prime}$, and the desired element is obtained by taking the inequalities $(e)$ presented above as equalities whenever $(k, i),(k, i+1)$ lie in the same $G$ orbit, and as strict inequalities if they lie in different orbits. Thus we have the following result.

Corollary 5.3. Let $z \in \mathbb{D}$ and let $G=\left(S_{\pi}\right)_{z}$. Then there exists $z^{\prime} \in \operatorname{ess}(\bar{v})$ such that $\left(S_{\pi}\right)_{z^{\prime}}=G$. Thus $\operatorname{dim} V_{\mathrm{soc}}\left[\bar{v}+z^{\prime}\right]=\frac{\left|S_{\pi}\right|}{|G|}$ and the FO inequality is sharp for all parabolic subgroups of $S_{\pi}$.

In the next section we will show that in the case were $\bar{v}=\mathbf{0}$ this bound can be obtained when $M$ a simple Verma module.

We conclude this section with the observation that Corollary $5 \cdot 3$ implies that if the essential support of a simple Gelfand-Tsetlin module intersects $\operatorname{ess}(\bar{v})$, then it must be isomorphic to soc $V(T(\bar{v}))$.

Corollary 5.4. If $z \in \operatorname{ess}(\bar{v})$ then the module $V_{\mathrm{soc}}=U D_{e}(\bar{v})$ is, up to isomorphism, the unique simple Gelfand-Tsetlin module having $\bar{v}+z$ in its support.

Proof. Let $\mathfrak{m}$ be the maximal ideal of $\Gamma$ corresponding to $\bar{v}$ and let $N=$ $U / U \mathfrak{m}$. Then being a simple module, $V_{\mathrm{soc}}$ appears as a quotient of $N$. By [FO14, Theorem 4.12(c)], $\operatorname{dim} N[\bar{v}+z] \leq \frac{\left|S_{\pi}\right|}{\left.\mid\left(S_{\pi}\right)_{z}\right) \mid}$, while $\operatorname{dim} V_{\mathrm{soc}}[\bar{v}+z]=$ $\frac{\left|S_{\pi}\right|}{\left.\mid\left(S_{\pi}\right)_{z}\right) \mid}$ for all $z \in \operatorname{ess}(\bar{v})$. This implies the corollary.

\section{Realization of Verma modules in universal tableaux modules}

Let $\lambda \in \mathbb{C}^{n}$ be a (Cartan) weight of $\mathfrak{g l}(n, \mathbb{C})$. Recall that we can associate to $\lambda$ a Verma module $M(\lambda)$ by extending $\lambda$ to a character of the Borel subalgebra of upper-triangular matrices and then inducing the resulting module to $\mathfrak{g l}(n, \mathbb{C})$. The module $M(\lambda)$ is a highest weight module with highest weight $\lambda$ and any other such module is a quotient of $M(\lambda)$.

6.1. Restriction from $\mathfrak{g l}(n, \mathbb{C})$ to $\mathfrak{s l}(n, \mathbb{C})$. We fix $\mathfrak{h}^{\prime} \subset \mathfrak{s l}(n, \mathbb{C})$ to be the usual Cartan subalgebra of diagonal matrices of trace 0 .

Given a representation $V$ of $\mathfrak{g l}(n, \mathbb{C})$ we denote by $V^{\prime}$ its restriction to $\mathfrak{s l}(n, \mathbb{C})$, and given $v \in V$ we denote by $v^{\prime}$ the corresponding vector in $V^{\prime}$. Then $v$ is a Cartan weight vector if and only if $v^{\prime}$ is a Cartan weight 
vector, and if $\lambda=\left(\lambda_{1}, \ldots, \lambda_{n}\right)$ is the weight of $v$ then the weight of $v^{\prime}$ is $\lambda^{\prime}=\left(\lambda_{1}-\lambda_{2}, \ldots, \lambda_{n-1}-\lambda_{n}\right)$. Verma modules restrict to Verma modules, and $M(\lambda)^{\prime}=M\left(\lambda^{\prime}\right)$. In particular this implies that $M(\lambda)$ is a free $U^{-}$module, where $U^{-} \subset U$ is the subalgebra generated by the elements $E_{j, i}$ with $1 \leq i<j \leq n$. We will say that $\lambda$ is dominant, resp. integral, resp. dominant integral, if the corresponding $\mathfrak{s l}(n, \mathbb{C})$-weight is dominant, resp. integral, resp. dominant integral.

The natural action of $S_{n}$ on $\mathbb{C}^{n}$ induces an action of $S_{n}$ on $\mathbb{C}^{n} / L$, where $L$ is the vector space generated by the vector $(1,1, \ldots, 1)$. If we identify $\mathbb{C}^{n} / L$ with the dual of the Cartan subalgebra of $\mathfrak{s l}(n, \mathbb{C})$ and the corresponding Weyl group with $S_{n}$, then the induced action of $S_{n}$ and the action of the Weyl group coincide. In other words, for each $\sigma \in S_{n}$ and each $\mathfrak{g l}(n, \mathbb{C})$ weight $\lambda$ we have $\sigma(\lambda)^{\prime}=\sigma\left(\lambda^{\prime}\right)$.

Let $\tilde{\rho}=-(0,1, \ldots, n-1)$. Then $\tilde{\rho}^{\prime}$ is the half-sum of the positive roots of $\mathfrak{s l}(n, \mathbb{C})$. The dot action of $S_{n}$ on $\mathbb{C}^{n}$ is given by $\sigma \cdot \lambda=\sigma(\lambda+\tilde{\rho})-\tilde{\rho}$ for all $\sigma \in S_{n}$. This dot action induces the dot action of $S_{n}$ on $\mathfrak{s l}(n, \mathbb{C})$-weights, i.e. $(\sigma \cdot \lambda)^{\prime}=\sigma \cdot \lambda^{\prime}$.

6.2. Highest weight vectors of universal tableaux modules. For each $\lambda \in$ $\mathbb{C}^{n}$ we set $\tilde{\lambda}=\lambda+\tilde{\rho}$.

Let $\lambda \in \mathbb{C}^{n}$ be such that $\tilde{\lambda}$ is a dominant weight, and let $\sigma \in S_{n}$. We denote by $H W(\lambda, \sigma)$ an element of $\mathbb{C}^{\mu}$ in normal form whose $k$-th row is a permutation of $\left(\tilde{\lambda}_{\sigma^{-1}(1)}, \tilde{\lambda}_{\sigma^{-1}(2)}, \ldots, \tilde{\lambda}_{\sigma^{-1}(k)}\right)$ for each $k$; this element may not be unique, so we fix one for each choice of $\lambda$ and $\sigma$. If $\tilde{\lambda}$ is dominant integral then the top row of $H W(\lambda, \sigma)$ is equal to $\tilde{\lambda}$.

Let $\tau \in S_{n}$. If $\sigma(\lambda)-\tau(\lambda) \in \mathbb{Z}^{n}$ then we can and will assume that $H W(\lambda, \sigma)$ and $H W(\lambda, \tau)$ lie in the same $\left(\mathbb{Z}_{0}^{\mu} \# S_{\mu}\right)$-orbit.

Lemma 6.1. Let $\bar{v}$ be a seed in the $\left(\mathbb{Z}_{0}^{\mu} \# S_{\mu}\right)$-orbit of $H W(\lambda, \sigma)$. Then the classical tableau $D_{e}(H W(\lambda, \sigma)) \in V(T(\bar{v}))$ is a highest weight vector of $V(T(\bar{v}))$ of weight $\sigma \cdot \lambda=\sigma(\lambda+\tilde{\rho})-\tilde{\rho}$.

Proof. Put $v=H W(\lambda, \sigma)$ and let $k \in \llbracket n \rrbracket$. The fact that $D_{e}(v)$ has the desired weight follows from the definition of $v$ and the formula for the action of $E_{k, k}$. It remains to prove that $D_{e}(v)$ is a highest weight vector.

Theorem 3.3 implies that $E_{k, k+1} D_{e}(v)$ is a linear combination of derivative tableaux $D_{\tau}\left(v+\delta^{k, i}\right)$ where $(k, i)$ is the first entry in an interval $I=\{(k, i),(k, i+1), \ldots,(k, j)\}$ and $\tau<\alpha(I)$. Furthermore, the coefficient of $D_{\tau}\left(v+\delta^{k, i}\right)$ is $\mathfrak{D}_{\tau, \alpha(I)}\left(e_{I}\right)(v)$. To prove $E_{k, k+1} D_{e}(v)=0$ it is enough to show that $\mathfrak{D}_{\tau, \alpha(I)}\left(e_{I}\right)(v)=0$ for all such $\tau$.

By definition, the interval $I$ is such that $v_{k, i}=v_{k, i+1}=\ldots=v_{k, j}$, and since the $k$-th row of $v$ is obtained by deleting one element from its $k+$ 1-th row, there are at least $|I|$ entries in the $k+1$-th row of $v$ equal to $v_{k, i}$. Thus $e_{I}$ is a rational function with a zero of order at least $|I|$ in $v$. 
On the other hand, by definition $\mathfrak{D}_{\tau, \alpha(I)}$ is a differential operator of order $\ell(\alpha(I))-\ell(\tau)<|I|$, and hence $\mathfrak{D}_{\tau, \alpha(I)}\left(e_{I}\right)(v)=0$.

We fix a seed $\bar{v}$ in the $\left(\mathbb{Z}_{0}^{\mu} \# S_{\mu}\right)$-orbit of $\operatorname{HW}(\lambda, \sigma)$ (again, the top row of $\bar{v}$ equals $\tilde{\lambda}$ if this is dominant integral). The lemma and the universal property of Verma modules together imply that there is a map $j_{\lambda, \sigma}: M(\sigma$. $\lambda) \rightarrow V(T(\bar{v}))$ mapping a highest weight vector $v_{\sigma \cdot \lambda}$ to $D_{e}(H W(\lambda, \sigma))$ for each $\sigma \in S_{n}$. As the following theorem shows, this map is injective and, therefore, any Verma module can be realized as a submodule of an adequate universal tableaux module.

Theorem 6.2. For each dominant weight $\lambda$ and each $\sigma \in S_{n}$ the map $j_{\lambda, \sigma}$ is injective. In particular every Verma module can be realized as a submodule of a universal tableaux module.

Proof. By the classical Verma and BGG theorems, the Verma module $M(\sigma$. $\lambda)^{\prime}$ has a simple socle, which is isomorphic to $M\left(v^{\prime}\right)$, where $v$ is the unique element in the orbit of $S_{n} \cdot \lambda$ such that $v^{\prime}$ is both linked to $\sigma \cdot \lambda^{\prime}$ and antidominant (see for example [Humo8, Chapter 4]). These conditions imply that $v=H W(\lambda, \sigma)$ and $w=H W(\lambda, v)$ lie in the same $\mathbb{Z}_{0}^{\mu} \# S_{\mu}$-orbit. In particular, they are both highest weight vectors in $V(T(\bar{v}))$ by Lemma 6.1.

Since $M(v)$ is simple and $j_{\lambda, v}$ is nonzero, it defines an isomorphism $M(v) \cong U D_{e}(w)$, and since the socle of $V(T(\bar{v}))$ is simple, it must be equal to $U D_{e}(w)$. By Proposition $4.4(\mathrm{~b})$ the socle of $V(T(\bar{v}))$ is contained in $U D_{e}(v)$, so the image of $j_{\lambda, \sigma}$ is a quotient of $M(\sigma \cdot \lambda)$ containing a highest weight vector of weight $v \cdot \lambda$. By [Humo8, Theorem 7.16] this is possible only if the map $j_{\lambda, \sigma}$ is injective.

6.3. The support of the Verma module $M(-\tilde{\rho})$. It follows from Lemma 6.1 that the tableau $D_{e}(\mathbf{0})$ is a highest weight vector of weight $-\tilde{\rho}$ in $V(T(\mathbf{0}))$. Since the Verma module $M(-\tilde{\rho})$ is simple, by Theorem 6.2 it is isomorphic to the socle of the corresponding universal tableaux module. This allows us to study both its Gelfand-Tsetlin support and its essential support. We begin our study of the support of $M(-\tilde{\rho})$ with the following lemma.

Lemma 6.3. Let $z \in \mathbb{D}(\mathbf{0})$, let $I=\llbracket a, b \rrbracket_{k} \in \mathbb{I}(\mathbf{0}, z)$, let $m=z_{k, a}=z_{k, a+1}=$ $\cdots=z_{k, b}$, and put $z^{\prime}=z-\delta^{k, b}$. Also, let $J$ be the (possibly empty) interval formed by the $(k-1, j) \in \Sigma$ such that $z_{k-1, j}=m$. Then $p_{z^{\prime}}\left(E_{k+1, k} D_{e}(z)\right)$ is nonzero if and only if $|J| \leq|I|$.

Proof. Recall that $\beta=\beta(I)$ is the permutation $(a a+1 \cdots b)_{(k)}$. The formulas for the action of $U$ on $V(T(\mathbf{0}))$ given in Theorem $3 \cdot 3$ tell us that

$$
p_{z^{\prime}}\left(E_{k+1, k} D_{e}(z)\right)=\sum_{\tau \leq \beta} \mathfrak{D}_{\tau, \beta}\left(f_{I}\right)(z) D_{\tau}\left(z^{\prime}\right) .
$$

The differential operator $\mathfrak{D}_{\tau, \beta}$ has order $\ell(\beta)-\ell(\tau) \leq \ell(\beta)=|I|$, and by definition $f_{I}$ has a zero of order $|J|$ at $z$. Thus $\mathfrak{D}_{\tau, \beta(I)}\left(f_{I}\right)(z)=0$ if $|J|>|I|$. 
Now suppose $|J| \leq|I|$ and let $\sigma=(a+|J| a+|J|+1 \cdots b)_{(k)}$. Then the order of $\mathfrak{D}=\mathfrak{D}_{\sigma, \beta}$ is $\ell(\beta)-\ell(\sigma)=|J|$. If we denote by $\partial_{k, i}$ the partial derivative with respect to $x_{k, i}$ then $\mathfrak{D}$ is a homogeneous polynomial of degree $|J|$ in $\partial_{k, i}$ with $a \leq i \leq b$. Using the definition of Stanley-Postnikov differential operators from [FGRZ18, paragraph 3.3], we see that the coefficient of $\partial_{k, b}^{|J|}$ in $\mathfrak{D}$ is precisely $\frac{(-1)^{|J|}}{|J| !}$. On the other hand, since $f_{I}$ is a rational function on the variables $x_{k, b}$ and $x_{k-1, j}$ with $(k-1, j) \in J$, we see that $\mathfrak{D}\left(f_{I}\right)=\frac{(-1)^{|J|}}{|J| !} \partial_{k, b}^{|J|}\left(f_{I}\right)$. Thus

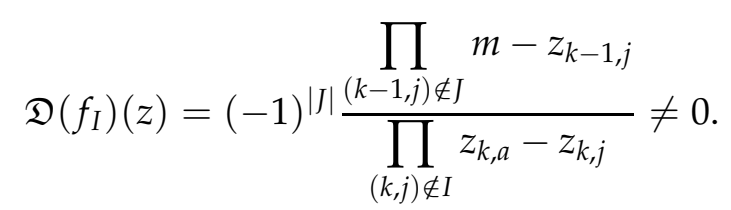

Since this is the coefficient of $D_{\sigma}\left(z^{\prime}\right)$ in $E_{k+1, k} D_{e}(z)$, we are done.

From this point on we identify $M(-\tilde{\rho})$ with $U D_{e}(\mathbf{0})$. We have already presented the graph $\widetilde{\Omega}(\mathbf{0})$ for $n=4$ in Example 4.2. In general, its edges are those of the form $[k, i] \rightarrow[k, i+1]$ for $1 \leq i \leq k-1$ and $[k, k] \rightarrow$ $[k-1,1]$ for all $2 \leq k \leq n$.

Let $v \in \mathbb{Z}_{0}^{\mu}$ be the element with $v_{k, i}=-i+1$ for all $1 \leq i \leq k \leq n-1$. For example, for $n=5$ we have

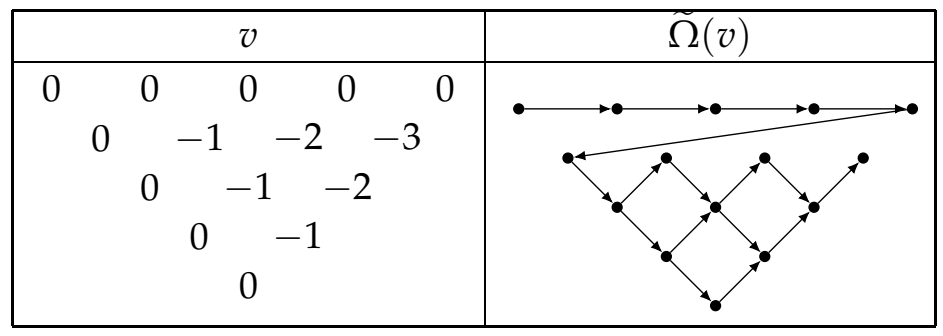

The edges of $\Omega^{+}(v)$ are precisely those of the form $[n, i]-[n-1, j]$ for all $i \in \llbracket n \rrbracket, j \in \llbracket n-1 \rrbracket$, along with all $[k, i]-[k-1, j]$ for $1 \leq i \leq j \leq k \leq n-1$. Recall from 85.1 that $\overline{\mathcal{P}}\left(\Omega^{+}(v)\right)$ is the set of those $z \in \mathbb{D}(\mathbf{0})$ such that $\Omega^{+}(v) \subset \Omega^{+}(z)$. We will prove that the support of $M(-\rho)$ is equal to the set of integral points $\overline{\mathcal{P}}\left(\Omega^{+}(v)\right)$.

Theorem 6.4. Let $z \in \mathbb{D}(\mathbf{0})$. Then $z$ lies in the Gelfand-Tsetlin support of $M(-\rho)$ if and only if $z_{k-1, j} \leq z_{k, i} \leq 0$ for all $k \leq n-1$ and $i \leq j$. Equivalently, the support of $M(-\rho)$ coincides with $\overline{\mathcal{P}}\left(\Omega^{+}(v)\right)$.

Proof. We first show that $\overline{\mathcal{P}}\left(\Omega^{+}(v)\right)$ is contained in the support of $M(-\tilde{\rho})$. By Lemma $4 \cdot 3$ it is enough to show that $D_{e}(v) \in M(-\tilde{\rho})$.

For each $l \in \llbracket n-1 \rrbracket$, let $v(l)$ be the element of $\mathbb{Z}_{0}^{\mu}$ such that $v(l)_{k, i}=$ $-i+1$ if $k \leq l$, while $v(l)_{k}=0$ if $k>l$; in other words, the $k$-th row of $v(l)$ is equal to that of $v$ if $k \leq l$ and equal to zero if $k>l$, in particular $v=$ 
$v(n-1)$. We now show that $v(l)$ lies in the support of $M(-\tilde{\rho})$ by induction on $l$, the case $v(1)=0$ being obvious. Suppose $v(l-1)$ lies in the support. Then row $l$ has exactly $l$ entries equal to 0 , while row $l-1$ has exactly one entry that equals 0 . If we apply Lemma $6.3 l-1$ times to rows $l$ and $l-1$ with $m=0$ we obtain that $v^{(1)}=v(l-1)-\left(\delta^{l, 2}+\delta^{l, 3}+\cdots+\delta^{l, l}\right)$ lies in the support of $M(-\tilde{\rho})$. Applying now the same lemma $l-2$ times to the same rows for $m=-1$, we see that $v^{(2)}=v^{(1)}-\left(\delta^{l, 3}+\cdots+\delta^{(l, l)}\right)$ also lies in the support of $M(-\tilde{\rho})$. Repeating this argument for $m=-2,-3, \cdots,-l+1$, we see that

$$
v^{(l-1)}=v(l-1)-\left(\delta^{2, l}+2 \delta^{3, l}+\cdots+(l-1) \delta^{l, l}\right)
$$

is in the support of $M(-\tilde{\rho})$. Thus $v=v(n-1)$ lies in the support.

We now prove that the support of $M(-\tilde{\rho})$ is contained in $\mathcal{P}\left(\Omega^{+}(v)\right)$. Suppose there exists $z$ in the support of $M(-\tilde{\rho})$ outside of $\overline{\mathcal{P}}\left(\Omega^{+}(v)\right)$, that is, that there exists some $j \leq i$ such that $z_{k, i}>z_{k+1, j}$. Since $z \in \mathbb{D}(\mathbf{0})$ we must have $z_{k+1, j} \geq z_{k+1, i}$, and hence $z_{k, i}>z_{k+1, l}$ for all $l \geq i$. We now show that we can assume $i=k$.

Suppose $i<k$ and let $w \in \mathbb{Z}_{0}^{\mu}$ be the element given by

$$
w_{l}= \begin{cases}\left(0^{(l)}\right) & k+2 \leq l \leq n \\ \left(0^{(i-1)},-1^{(k-i+2)}\right) & l=k+1 \\ \left(0^{(i)},-1^{(k-i)}\right) & l=k \\ \left(-1^{(l)}\right) & 1 \leq l \leq k-1\end{cases}
$$

where $m^{(j)}$ denotes a sequence of $j$ consecutive entries equal to $m$. Then $\Omega^{-}(w) \subset \Omega^{-}(z)$ and hence by Lemma 4.3 we have $D_{e}(w) \in M(-\tilde{\rho})$. Applying Lemma 6.3 twice to rows $k+1, k$ with $m=-1$, we see that $z^{\prime}=w-$ $\delta^{k+1, k+1}-\delta^{k+1, k}$ also belongs to the support of $M(-\tilde{\rho})$. Direct inspection shows that $\Omega^{-}\left(-\delta^{k+1, k}-\delta^{k+1, k+1}\right) \subset \Omega^{-}\left(w^{\prime}\right)$, so $z^{\prime \prime}=-\delta^{k+1, k}-\delta^{k+1, k+1}$ also lies in the support of $M(-\tilde{\rho})$, or equivalently $D_{e}\left(z^{\prime}\right) \in M(-\tilde{\rho})$.

Now any element in the Gelfand-Tsetlin component of weight $z^{\prime \prime}$ has Cartan weight $\tilde{\rho}-2 \alpha$, where $\alpha$ is the Cartan weight of $E_{k+2, k+1}$. The only such elements in $M(-\tilde{\rho})$ are the scalar multiples of $E_{k+2, k+1}^{2} D_{e}(\mathbf{0})$. Applying Lemma 6.3 twice to $D_{e}(\mathbf{0})$ we see that the projection of this element to the $z^{\prime \prime}$-Gelfand-Tsetlin weight component is zero. This is a contradiction, which arose from assuming the existence of $z$ in the support of $M(-\tilde{\rho})$ but not in $\overline{\mathcal{P}}\left(\Omega^{+}(v)\right)$.

6.4. The essential support of $M(-\tilde{\rho})$. Let $w \in \mathbb{D}(\mathbf{0})$ be the element given by $w_{n, i}=0$ and $w_{k, i}=w_{k+1, k+1}-i+1$ for all $k \leq n$. For example if $n=5$ then 


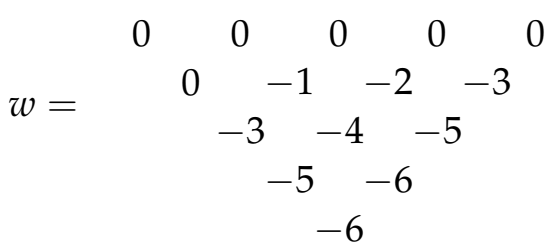

Clearly, $\Omega^{+}(w)=\Omega^{+}(\mathbf{0})$ and hence

$$
\operatorname{dim} M(-\tilde{\rho})[w]=\left|S_{\pi}\right|=(n-1) !(n-2) ! \cdots 2 ! 1 !,
$$

thus showing $w$ lies in the essential support of $D_{e}(\mathbf{0})$. In fact, we have even a stronger statement, namely, that the essential support of $M(-\tilde{\rho})$ is equal to the rational cone $\overline{\mathcal{P}}\left(\Omega^{+}(w)\right)$. As in our study of the support, we need a preliminary lemma; again, we denote by $m^{(j)}$ a sequence of $j$ consecutive entries equal to $m$.

Lemma 6.5. Let $1 \leq s<k \leq n-2$, and let $z \in \mathbb{D}(0)$ be such that $z_{k+1}=$ $\left(0^{(k)},-1\right)$ and $z_{k}=\left(0^{(s-1)},-1^{(k-s+1)}\right)$. If $z$ lies in the essential support of $M(-\tilde{\rho})$ then so does $z+\delta^{k, s}$.

Proof. Set $z^{\prime}=z+\delta^{k, s}$, and set $I=\llbracket s+1, k \rrbracket_{(k)}$. We put $\omega=\omega_{0}^{z^{\prime}}$ and $\sigma=\omega(s s+1)_{(k)} \beta(I)$. An explicit calculation shows that

$$
\sigma_{(k)}(i)= \begin{cases}i+k-s-1 & \text { if } 1 \leq i<s \\ k & \text { if } i=s \\ i-s & \text { if } s<i<k \\ k-1 & \text { if } i=k\end{cases}
$$

while $\sigma_{(l)}=\omega_{(l)}$ for $l \neq k$, so $\sigma$ is a $z$-shuffle. By definition, $\sigma \alpha(I)=$ $\omega(s s+1)$, thus $\mathfrak{D}_{\omega, \sigma \alpha(I)}=\partial_{k, s}-\partial_{k, s+1}$.

Since $z$ lies in the essential support of $M(-\tilde{\rho})$, we know that $D_{\sigma}(z) \in$ $M(-\tilde{\rho})$. Using the formulas in Theorem 3.3. we see that the coefficient of $D_{\omega}\left(z^{\prime}\right)$ in $E_{k, k+1} D_{\sigma}(z)$ is $\left(\partial_{k, s}-\partial_{k, s+1}\right)\left(e_{I}\right)$ evaluated at $z$. Putting $\tilde{e}_{I}=$ $\frac{e_{I}}{x_{k, s}-x_{k+1, k+1}}$ then

$$
\mathfrak{D}_{\omega, \sigma \alpha(I)}\left(e_{I}\right)=\left(x_{k, s}-x_{k+1, k+1}\right)\left(\partial_{k, s}-\partial_{k, s+1}\right)\left(\tilde{e}_{I}\right)-\tilde{e}_{I},
$$

which evaluates to $(-1)^{k-s+1}$ at $z$. By Proposition $3 \cdot 5(\underline{b}) p_{z^{\prime}}\left(E_{k, k+1} D_{\sigma}(z)\right)$ generates $V(T(\mathbf{0}))\left[z^{\prime}\right]$ and hence $z^{\prime}$ lies in the essential support of $M(-\tilde{\rho})$.

Theorem 6.6. Let $(k, i) \in \Sigma^{\prime}$, and denote by $c^{k, i}$ the element in $\mathbb{Z}_{0}^{\mu}$ for which $c_{l, j}^{k, i}$ is 0 if $l>k$ or if $l=k$ and $i<j$, and -1 otherwise. Then $\mathcal{P}\left(\Omega^{+}(\mathbf{0})\right)=$ $\sum_{1 \leq i \leq k \leq n-1} \mathbb{N} c^{k, i}$, and $\mathcal{P}\left(\Omega^{+}(\mathbf{0})\right)$ coincides with the essential support of $M(-\tilde{\rho})$. Proof. The description of $\mathcal{P}\left(\Omega^{+}(\mathbf{0})\right)$ given in the proof of Theorem 5.2 , so we just have to show that it is equal to the essential support of $M(-\tilde{\rho})$. 
We note first that the edges of $\Omega=\Omega^{+}(\mathbf{0})$ are the edges of the form $[k+$ $1, j]-[k, i]$ for $j \in \llbracket k+1 \rrbracket$ and $i \in \llbracket k \rrbracket$. Take $z \in \mathbb{D}(\mathbf{0})$. Then $z$ lies outside $\mathcal{P}(\Omega)$ if and only if $\Omega^{-}(z)$ has at least one edge, say $[k, i]-[k+1, j]$, or equivalently if and only if $z_{k, i}>z_{k+1, j}$. Since $z_{k, i} \leq 0$ for all $k \leq n-1$, we must have $k+1<n$. Furthermore, since $z$ is in normal form we have $z_{k, l} \geq z_{k, i}>z_{k+1, k+1}$, so every edge in $\mathcal{V}(k, i)=\{[k, l]-[k+1, k+1] \mid l \leq i\}$ is an edge of $\Omega^{-}(z)$. By Lemma 4.3 if $z$ is in the essential support of $M(-\tilde{\rho})$ then so is any element $w$ such that the edges of $\Omega^{-}(w)$ are contained in $\mathcal{V}(k, i)$.

Assume there is an element $z \in \mathbb{D}(\mathbf{0})$ which is in the essential support of $M(-\tilde{\rho})$ but not in $\mathcal{P}(\Omega)$ and let $k$ be minimal such that some $\mathcal{V}(k, i)$ is contained in the edge set of $\Omega^{-}(z)$. Suppose first that $k=1$. Then $\mathcal{V}(1,1)$ is the set of edges of $\Omega^{-}\left(-\delta^{2,2}\right)$, and hence $-\delta^{2,2}$ lies in the essential support of $M(-\tilde{\rho})$ and the Gelfand-Tsetlin component of this weight has dimension 2. The Cartan weight of any element in this component is $-\alpha-\tilde{\rho}$, where $\alpha$ is the Cartan weight of $E_{2,3}$, and since $M(-\tilde{\rho})$ is a free $U^{-}$-module and a highest weight module with highest weight $-\tilde{\rho}$, it contains only one element with this weight, namely $E_{3,2} D_{e}(\mathbf{0})$, a contradiction. Hence $k \geq 2$.

Let $(k, s) \in \Sigma$ and denote by $z(k, s)$ the element given by

$$
z(k, s)_{l}= \begin{cases}\left(0^{(l)}\right) & \text { for } k+2 \leq l \leq n \\ \left(0^{(k)},-1\right) & \text { for } l=k+1 \\ \left(0^{(s)},-1^{(k-s)}\right) & \text { for } l=k \\ \left(-1^{(l)}\right) & \text { for } 1 \leq l \leq k-1\end{cases}
$$

Applying $k-s$ times Lemma 6.5 to rows $k, k+1$ we see that $z(k, k-1)$ also lies in the essential support of $M(-\tilde{\rho})$. Now if $k>2$ then we can also apply Lemma 6.5 to $z(k, k-1)$ in rows $k-1, k$ to obtain an element $z^{\prime}$ in the essential support of $M(-\tilde{\rho})$ such that $z_{k-1,1}^{\prime}>z_{k, k^{\prime}}^{\prime}$ contradicting the minimality of $k$. Thus we must have $k=2$ and $z(2,1)=-\delta^{3,3}-\delta^{2,2}-\delta^{1,1}$ must lie in the essential support of $M(-\tilde{\rho})$. In particular this implies that the component of Gelfand-Tsetlin weight $z(2,1)$ of $M(-\tilde{\rho})$ must be of dimension 6.

On the other hand, any vector of Gelfand-Tsetlin weight $z(2,1)$ must have Cartan weight $-\alpha_{1,4}-\tilde{\rho}$, where $\alpha_{1,4}$ is the Cartan weight of $E_{1,2} E_{2,3} E_{3,4}$. However the space of weight $-\alpha_{1,4}$ in $U^{-}$is four dimensional, which implies that the dimension of the Gelfand-Tsetlin component of weight $z(2,1)$ of $M(-\tilde{\rho})$ can be at most four, a contradiction.

Example 6.7. If $n=4$ then the support of $M(-\tilde{\rho})$ consists of all tableaux 


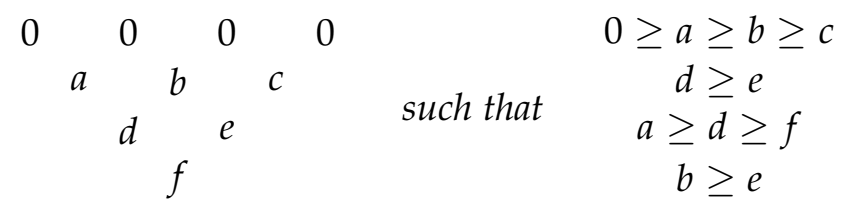

On the other hand, the essential support of $M(-\tilde{\rho})$ consists of those tableaux such that $0 \geq a \geq b \geq c \geq d \geq e \geq f$.

We conclude this paper with the observation that the simple Verma modules of $\mathfrak{g l}(n, \mathbb{C})(n \geq 4)$, considered as Gelfand-Tsetlin modules, do not have necessarily a basis of derivative tableaux.

Remark 6.8. Let $z=-\delta^{1,1}-\delta^{2,2}-\delta^{3,3}$. A consecutive application of the Gelfand-Tsetlin formulas shows that

$$
\begin{gathered}
E_{4,3} E_{3,2} E_{2,1} D_{e}(\mathbf{0})=-D_{e}(z)+D_{(32)_{(3)}}(z)-D_{(123)_{(3)}}(z)+ \\
D_{(12)_{(2)}}(z)-D_{(12)_{(2)}(32)_{(3)}}(z) .
\end{gathered}
$$

As mentioned above, the Gelfand-Tsetlin component of $M(-\tilde{\rho})$ of weight $z$ has dimension at most 4. Hence the Verma module $M(-\tilde{\rho})$ does not have a basis formed by derivative tableaux. It is an interesting open question to find a basis of the Verma modules, even of $M(-\tilde{\rho})$, inside $V(T(\bar{v}))$.

\section{References}

[BBo5] A. Björner and F. Brenti, Combinatorics of Coxeter groups, Graduate Texts in Mathematics, vol. 231, Springer, New York, 2005.

[EMV] N. Early, V. Mazorchuk, and E. Vyshniakova, Canonical Gelfand-Zeitlin modules over orthogonal Gelfand-Zeitlin algebras. Preprint, available online at https://arxiv.org/abs/1709.01553

[FGR15] V. Futorny, D. Grantcharov, and L. E. Ramirez, Irreducible Generic Gelfand-Tsetlin modules of $\mathfrak{g l}(n)$, SIGMA 18 (2015).

[FGR16] _ Singular Gelfand-Tsetlin modules of $\mathfrak{g l}(n)$, Adv. Math. 290 (2016), 453482.

[FGR17a] _ New Singular Gelfand-Tsetlin modules of $\mathfrak{g l}(n)$ of index 2, Comm. Math. Phys. 355 (2017), no. 3, 1209-1241.

[FGR17b] - Drinfeld category and the classification of singular GelfandTsetlin $\mathfrak{g l}_{n}$-modules, Int. Math. Res. Not. (2017), rnx159, available at /oup/backfile/content_public/journal/imrn/pap/10.1093_imrn_rnx159/1/rnx159.pdf

[FGRZ18] V. Futorny, D. Grantcharov, L. E. Ramirez, and P. Zadunaisky, GelfandTsetlin theory for rational Galois algebras (2018). Preprint, available online at https://arxiv.org/abs/1801.09316

[FO14] V. Futorny and S. Ovsienko, Fibers of characters in Gelfand-Tsetlin categories, Trans. Amer. Math. Soc. 366 (2014), no. 8, 4173-4208.

[FRZ19] V. Futorny, L. E. Ramirez, and J. Zhang, Combinatorial construction of GelfandTsetlin modules for $\mathfrak{g l}_{n}$, Adv. Math. 343 (2019), 681-711.

[Har17] J. Hartwig, Principal Galois Orders and Gelfand-Zeitlin modules (2017). Preprint, available online at https://arxiv.org/abs/1710.04186 
[Humo8] J. E. Humphreys, Representations of semisimple Lie algebras in the BGG category $\mathcal{O}$, Graduate Studies in Mathematics, vol. 94, American Mathematical Society, Providence, RI, 2008.

$\left[\mathrm{KTW}^{+}{ }_{18}\right.$ 8] J. Kamnitzer, P. Tingley, B. Webster, A. Weekes, and O. Yacobi, On category $\mathcal{O}$ for affine Grassmannian slices and categorified tensor products (2018). Preprint, available online at https://arxiv.org/abs/1806.07519

[MV] V. Mazorchuk and E. Vyshniakova, Harish-Chandra modules over invariant subalgebras in a skew-group ring. Preprint, available online at https://arxiv.org/abs/1811.00332

[RZ18] L. E. Ramírez and P. Zadunaisky, Gelfand-Tsetlin modules over $\mathfrak{g l}(n)$ with arbitrary characters, J. Algebra 502 (2018), 328-346.

[Vis] E. Vishniakova, Geometric approach to p-singular Gelfand-Tsetlin $\mathfrak{g l}(n)$-modules. Preprint, available online at https://arxiv.org/abs/1705.05793

[Vis18] E. Vishnyakova, A Geometric approach to 1-singular Gelfand-Tsetlin $\mathfrak{g l}_{n}$-modules, Differ. Geom. Appl. 56 (2018), 155-160.

[Zad17] P. Zadunaisky, A new way to construct 1-singular Gelfand-Tsetlin modules, Algebra Discrete Math. 23 (2017), no. 1, 180-193.

Instituto de Matemática e Estatística, Universidade de São Paulo, São Paulo SP, BRASIL

E-mail address: futorny@ime.usp.br,

University of Texas at Arlington, Arlington, TX 76019, USA

E-mail address: grandim@uta.edu

Universidade Federal do ABC, SANTo ANdRÉ-SP, Brasil

E-mail address: luis.enrique@ufabc.edu.br,

Universidad CAECE and Universidad de Buenos Aires - Buenos Aires, Argentina

E-mail address: pzadunaiskybustillos@caece.edu.ar 